\title{
Prompt atmospheric neutrinos in the quark-gluon string model
}

\author{
S. I. Sinegovsky ${ }^{1,2, a}$, M. N. Sorokovikov ${ }^{1,2, b}$ \\ ${ }^{1}$ Joint Institute for Nuclear Research, Joliot-Curie, 6, Dubna, Moscow region 141980, Russia \\ ${ }^{2}$ Irkutsk State University, Gagarin blv. 20, Irkutsk 664003, Russia
}

Received: 28 February 2019 / Accepted: 8 December 2019 / Published online: 15 January 2020

(C) The Author(s) 2020

\begin{abstract}
We calculate the atmospheric flux of prompt neutrinos, produced in decays of the charmed particles at energies beyond $1 \mathrm{TeV}$. Cross sections of the $D$ mesons and $\Lambda_{c}^{+}$ baryons production in $p A$ and $\pi A$ collisions are calculated in the phenomenological quark-gluon string model (QGSM) which is updated using recent measurements of cross sections of the charmed meson production in the LHC experiments. A new estimate of the prompt atmospheric neutrino flux is obtained and compared with the limit from the IceCube experiment, and with predictions of other charm production models.
\end{abstract}

\section{Introduction}

At present time the operating neutrino telescopes focus on the detection of astrophysical high-energy neutrino fluxes: IceCube, a cubic kilometer detector at the South Pole [1$3]$, ANTARES $[4,5]$ located in the Mediterranean Sea, and underwater Baikal Gigaton Volume Detector (Baikal-GVD), a cubic kilometer-scale array, which is currently under construction in Lake Baikal [6,7].

The Baikal-GVD has a module structure and consists of functionally independent sub-arrays (clusters) of optical modules (OMs) and is designed to detect astrophysical neutrino fluxes at energies from a few $\mathrm{TeV}$ up to $100 \mathrm{PeV}$. Five clusters have been already installed (every one includes 288 $\mathrm{OMs}$ ), the current instrumented volume of the Baikal-GVD is the largest in the Northern Hemisphere $\left(\sim 0.25 \mathrm{~km}^{3}\right)$, and the first high-energy neutrino induced events are reconstructed. The first phase (GVD-1) to be completed by 2021 and will comprise nine clusters (2592 OMs), the full-scale GVD with an instrumented volume about of $2 \mathrm{~km}^{3}$ will consist of $10^{4}$ light sensors.

\footnotetext{
a e-mail: sinegovsky@jinr.ru

be-mail: sorokovikov@jinr.ru
}

The diffuse flux of high-energy astrophysical neutrinos was revealed in 2013 at IceCube detector [8,9], and for 6 years about 100 neutrino events were detected in the IceCube experiment [2]. Another important discovery was made recently: on 22 Sept. 2017 IceCube have detected the high-energy neutrino event coincident both in the direction and the time with the gamma-ray flare from the blazar TXS 0506+056 [10]. The event was later confirmed [11] by the archival IceCube data which display an excess of high-energy neutrino events (against the atmospheric neutrino flux) between Sept. 2014 and Mar. 2015, which gives $3.5 \sigma$-evidence for neutrino flux from the direction of TXS 0506+056. This supports the hypothesis that the blazar TXS $0506+056$ is the individual source of high-energy neutrinos and, presumably, the source of high-energy cosmic rays.

Essential progress has been achieved in experimental studies of astrophysical and atmospheric neutrino fluxes; however, the prompt atmospheric neutrinos have not yet been detected. High-energy neutrinos arising from decays of mesons and baryons, produced in hadronic collisions of cosmic rays with Earth's atmosphere, compose the background against the neutrinos from distant astrophysical sources. The atmospheric neutrinos comprise two components, which are distinguished by zenith-angle distributions and the energy spectra. The anisotropic component, arising from decays of pions and kaons, has the softer spectrum ("conventional" or $\pi, K$-neutrinos). The second component, quasi-isotropic flux produced at higher energies, mainly in decays of short-lived heavy charmed mesons and baryons $D, \Lambda_{c}^{+}$, is characterized by a harder spectrum. This component ("prompt" neutrinos) is most uncertain because of scarce measurements and wide spread in model predictions of the charm production cross sections at very high energies.

The high-energy interactions of cosmic rays with the Earth's atmosphere are dominated by the soft processes with small momentum transfer, which are beyond the scope of perturbative technique of the quantum chromodynamics (QCD). Perturbative QCD models of the charmed particle production 
encounter difficulties related to the nonperturbative dynamics contribution. Thus, the elaboration of phenomenological models beyond the $\mathrm{PQCD}$ is required for comprehensive research of the charm production in hadronic interactions at high energies.

The quark-gluon string model (QGSM) was developed [12-14] to describe the soft and semihard hadronic processes at high energies: it has been applied for successful explanation of characteristics of mesons and baryons production in hadron-nucleon collisions. QGSM was one of the first models to estimate the prompt atmospheric neutrino flux [15]. The recent data on the cross sections of charmed particle production, obtained in experiments at the LHC [16-19], allow the QGSM free parameters to be updated. The updated version of QGSM is applied to calculate the prompt neutrino flux in the neutrino energy range $1 \mathrm{TeV}-100 \mathrm{PeV}$. The calculation is based on the hadronic cascade model $[15,20,21]$ and cross sections of $D$ meson and $\Lambda_{c}^{+}$baryon production in $p A$ - and $\pi A$-collisions which are computed with the updated QGSM. We compare our result with the constraint obtained in the IceCube experiment [1] and with predictions of the color dipole model (ERS) [22], SIBYLL 2.3c [23], the NLO pQCD models, BEJKRSS [24] and GRRST [25].

\section{Production of charmed particles in QGSM}

The nonperturbative quark-gluon string model (QGSM) gives unified descriptions of the soft hadronic processes. The model is based on the reggeon calculus, the topological $1 / N_{\mathrm{c}}$-expansion of the amplitudes and the color string dynamics (see for more details [12,13,26-28] and the references therein). The QGSM, having a small number of parameters, succeeded in describing the multiparticle production in hadron-nucleus collisions at high energies [12-14,26-31].

Inelastic processes in the QGSM are described by the reggeon exchange (planar diagrams) with the intercept $\alpha_{\mathrm{R}}(0)<1$ and by the supercritical pomeron exchange (cylinder-type diagrams) with the intercept $\alpha_{P}(0)=1+\Delta$, where $\Delta>0$. At high energies, the contribution of cylindrical diagrams dominates due to the factor $\left(s / s_{0}\right)^{\Delta}$, whereas the contribution of planar diagrams decreases as $\left(s / s_{0}\right)^{\alpha_{\mathrm{R}}(0)-1} \propto$ $\left(s / s_{0}\right)^{-1 / 2}$.

The planar and cylinder-type diagrams involve quarkquark, quark-gluon and gluon-gluon scattering. The summation of $q q, q g$ and $g g$-diagrams leads to the Regge-behavior of the scattering amplitude. The $s$-channel cuttings of cylindric diagrams describe the multiparticle production, and in the $t$-channel, these diagrams correspond to gluon exchanges. It is argued [31] that cylindric diagrams lead to the pomeron pole as the color singlet is made up of sea quarks and soft gluons. The pomeron can be related to a sum of the ladder diagrams with exchange of reggeized gluons. A sum of gluon ladders with possible quark loop insertions may produce the pomeron trajectory. The simplest two-gluon exchange leads to a pomeron with the intercept $\alpha_{P}(0)=2 S_{\mathrm{g}}-1=1$. Taking account of mixing between $g g$ and $q \bar{q}$ Regge trajectories (glueballs and $q \bar{q}$ resonances) at the small $t$ and the effects of the small distance perturbative dynamics leads to the supercritical pomeron with $\Delta=\alpha_{P}(0)-1 \sim 0.15-0.25$, which ensures the Froissart behavior of the total cross section.

To calculate the inclusive cross sections of charmed hadron production, one needs to know the distribution functions of the quarks of the colliding particles and the fragmentation functions of the quarks and diquarks. The inclusive cross sections of charmed hadron production are defined as the convolution of distribution functions of the valence (and the sea) quarks and diquarks of the colliding particles with the functions of quarks (diquarks) fragmentation into a charmed hadron. These functions are expressed in terms of the intercept $\alpha_{\mathrm{R}}(0)$ of the Regge trajectory $\left(\alpha_{\mathrm{R}}(t) \simeq \alpha_{\mathrm{R}}(0)+\alpha_{\mathrm{R}}^{\prime} t\right.$ in linear approximation), including the $\alpha_{\psi}(t)$ trajectory of the $c \bar{c}$ bound states. The complete set of distributions and fragmentation functions can be found in Refs. [13,26-29].

For a nucleon target, the inclusive cross section of production of a hadron $h\left(h=D^{+}, D^{-}, D^{0}, \bar{D}^{0}, \Lambda_{c}^{+}\right)$can be written as a sum over $n$-pomeron cylinder diagrams:

$\tilde{x} \frac{\mathrm{d} \sigma}{\mathrm{d} x}=\int E \frac{\mathrm{d}^{3} \sigma}{\mathrm{d}^{3} p} \mathrm{~d}^{2} p_{\perp}=\sum_{n=0}^{\infty} \sigma_{n}(s) \varphi_{n}^{h}(s, x)$,

where $\sigma_{n}(s)$ is the cross section of the $2 n$-strings (chains) production, corresponding to the $s$-channel discontinuity of the multipomeron diagrams ( $n$ cut pomerons and arbitrary number of external pomerons taking part in the elastic rescattering); $\varphi_{n}^{h}(s, x)$ is the $x$-distribution of the hadron $h$ produced in the fission of $2 n$ quark-gluon strings: $\varphi_{0}^{h}(s, x)$ accounts for the contribution of the diffraction dissociation of colliding hadrons, $n=1$ corresponds to the strings formed by valence quarks and diquarks, terms with $n>1$ are related to sea quarks and antiquarks; $x=2 p_{\|} / \sqrt{s}$ is the Feynman variable, $p_{\|}$is the longitudinal momentum of the produced hadron, $\sqrt{s}$ is the total energy of the two colliding hadrons in the c.m.f., $\tilde{x}=\sqrt{x_{\perp}^{2}+x^{2}}, x_{\perp}=2 m_{\perp} / \sqrt{s}, m_{\perp}=\sqrt{<p_{\perp}^{2}>+m_{h}^{2}}$, $<p_{\perp}^{2}>$ is the mean square of the transverse momentum, and $m_{h}$ is the mass of the hadron $h$.

The cross sections $\sigma_{\mathrm{n}}(s)$ were calculated [32] in the quasieikonal approximation taking into account the lowmass diffractive excitations of the colliding particles and it corresponds to maximum inelastic diffraction consistent with the unitarity condition. Only nonenhanced graphs were considered with neglect of interactions between pomerons:

$\sigma_{\mathrm{n}}(s)=\frac{\sigma_{P}(s)}{n z(s)}\left[1-e^{-z(s)} \sum_{k=0}^{n-1} \frac{[z(s)]^{k}}{k !}\right], \quad(n \geq 1)$, 
where

$\sigma_{P}(s)=8 \pi \gamma_{P}\left(s / s_{0}\right)^{\Delta}, \quad z(s)=\frac{2 C \gamma_{P}\left(s / s_{0}\right)^{\Delta}}{R^{2}+\alpha_{P}^{\prime} \ln \left(s / s_{0}\right)}$.

Here $\sigma_{P}(s)$ is the pomeron contribution to the total cross section, $z(s)$ is the function representing the relative contribution of the successive rescatterings, $\gamma_{P}$ and $R^{2}$ are characteristics of the pomeron residue; $s_{0}=1 \mathrm{GeV}^{2}$. The term with $n=0$ in (1) corresponds to the elastic scattering and the diffractive dissociation: $\sigma_{0}(s)=\sigma_{\mathrm{el}}+\sigma_{\mathrm{DD}}=\sigma_{P}(s)[f(z / 2)-f(z)]$, where

$f(z(s))=\frac{1}{z(s)} \int_{0}^{z(s)} \frac{1-e^{-y}}{y} \mathrm{~d} y$.

The parameter $C=1+\sigma_{\mathrm{DD}} / \sigma_{\mathrm{el}}>1$ takes into account the low-mass diffractive dissociation.

The values of the above parameters are found from experimental data on the total and differential cross sections of elastic $p p$ and $p \bar{p}$ scattering at high energies [14,27,31,33]:

$$
\begin{aligned}
& \gamma_{P}^{p p}=1.27 \mathrm{GeV}^{-2}, R_{p p}^{2}=4.0 \mathrm{GeV}^{-2}, C_{p p}=1.8, \\
& \gamma_{P}^{\pi p}=1.07 \mathrm{GeV}^{-2}, R_{\pi p}^{2}=2.48 \mathrm{GeV}^{-2}, C_{\pi p}=1.65, \\
& \Delta=0.156, \alpha_{P}^{\prime}=0.25 \mathrm{GeV}^{-2} .
\end{aligned}
$$

In the case of $D$ meson production in the $p p$ interaction, the functions $\varphi_{n}^{h}(s, x)$ can be written [13] as follows:

$$
\begin{aligned}
& \varphi_{n}^{D}(s, x) \\
& =a^{D}\left\{F_{q_{V}}^{D(n)}\left(x_{+}\right) F_{q q}^{D(n)}\left(x_{-}\right)+F_{q q}^{D(n)}\left(x_{+}\right) F_{q_{V}}^{D(n)}\left(x_{-}\right)\right. \\
& \left.\quad+2(n-1) F_{q_{\mathrm{sea}}}^{D(n)}\left(x_{+}\right) F_{q_{\mathrm{sea}}}^{D(n)}\left(x_{-}\right)\right\},
\end{aligned}
$$

where $x_{ \pm}(s)=\frac{1}{2}\left[\sqrt{x^{2}+4 m_{\perp}^{2} / s} \pm x\right]$.

For the $\pi^{-} p$ interactions $[13,27]$

$$
\begin{aligned}
& \varphi_{n}^{D}(s, x) \\
& =a^{D}\left\{F_{\bar{q}_{V}}^{D(n)}\left(x_{+}\right) F_{q_{V}}^{D(n)}\left(x_{-}\right)+F_{q_{V}}^{D(n)}\left(x_{+}\right) F_{q q}^{D(n)}\left(x_{-}\right)\right. \\
& \left.\quad+2(n-1) F_{q_{\mathrm{sea}}}^{D(n)}\left(x_{+}\right) F_{q_{\mathrm{sea}}}^{D(n)}\left(x_{-}\right)\right\} .
\end{aligned}
$$

The functions $F_{q_{V}}^{D(n)}\left(x_{ \pm}\right), \quad F_{\bar{q}_{V}}^{D(n)}\left(x_{ \pm}\right), \quad F_{q q}^{D(n)}\left(x_{ \pm}\right)$, and $F_{q_{\text {sea }}}^{D(n)}\left(x_{ \pm}\right)$defined as convolution of the quark distributions with the fragmentation functions, take into account contributions of the valence quarks, diquarks, and sea quarks. For example, in the $p p$ collisions [26-28]:

$$
\begin{aligned}
F_{q_{V}}^{D(n)}\left(x_{ \pm}\right)= & \frac{2}{3} \int_{x_{ \pm}}^{1} f_{p}^{u_{V}(n)}\left(x_{1}\right) G_{u}^{D}\left(x_{ \pm} / x_{1}\right) \mathrm{d} x_{1} \\
& +\frac{1}{3} \int_{x_{ \pm}}^{1} f_{p}^{\mathrm{d}_{V}(n)}\left(x_{1}\right) G_{d}^{D}\left(x_{ \pm} / x_{1}\right) \mathrm{d} x_{1},
\end{aligned}
$$

$$
\begin{aligned}
F_{q q}^{D(n)}\left(x_{ \pm}\right)= & \frac{2}{3} \int_{x_{ \pm}}^{1} f_{p}^{u d(n)}\left(x_{1}\right) G_{u d}^{D}\left(x_{ \pm} / x_{1}\right) \mathrm{d} x_{1} \\
& +\frac{1}{3} \int_{x_{ \pm}}^{1} f_{p}^{u u(n)}\left(x_{1}\right) G_{u u}^{D}\left(x_{ \pm} / x_{1}\right) \mathrm{d} x_{1} .
\end{aligned}
$$

In the case of the $\pi^{-} p$ interactions $[27,28]$

$$
\begin{aligned}
& F_{q_{V}}^{D(n)}\left(x_{+}\right)=\int_{x_{+}}^{1} f_{\pi}^{d_{V}(n)}\left(x_{1}\right) G_{d}^{D}\left(x_{+} / x_{1}\right) \mathrm{d} x_{1}, \\
& F_{\bar{q}_{V}}^{D(n)}\left(x_{+}\right)=\int_{x_{+}}^{1} f_{\pi}^{\bar{u}_{V}(n)}\left(x_{1}\right) G_{\bar{u}}^{D}\left(x_{+} / x_{1}\right) \mathrm{d} x_{1},
\end{aligned}
$$

where $f_{p}^{j}(x), f_{\pi}^{j}(x)$ are the distribution functions of quarks, antiquarks and diquarks in colliding hadrons, $j=q, \bar{q}, q q$; $G_{j}^{D}\left(x / x_{1}\right)$ are the fragmentation functions. In the limits $x \rightarrow 0$ and $x \rightarrow 1$ these functions are defined by Regge asymptotics, and for the intermediate values of $x$ the interpolation is used $[13,26,27]$. In particular,

$$
\begin{aligned}
f_{p}^{u_{V}(n)}(x)= & C_{n}^{u_{V}} x^{-\alpha_{R}(0)}(1-x)^{\alpha_{R}(0)-2 \alpha_{N}(0)+n-1}, \\
G_{d}^{D^{-}}\left(x / x_{1}\right)= & G_{\bar{u}^{D}}^{D^{0}}\left(x / x_{1}\right)=\left(1-x / x_{1}\right)^{\lambda-\alpha_{\psi}(0)}[1 \\
& \left.+a_{1}\left(x / x_{1}\right)^{2}\right]
\end{aligned}
$$

where $\alpha_{R}(0)=0.5, \alpha_{N}(0)=-0.5, \alpha_{\psi}(0)=-2.2, \lambda=$ $2<p_{\perp}^{2}>\alpha_{R}^{\prime}=0.5$, and the coefficient $C_{n}^{u_{V}}$ is determined by normalization $\int_{0}^{1} f_{p}^{u_{V}(n)}(x) \mathrm{d} x=1$. More details on the functions $\varphi_{n}^{h}(s, x), f_{p}^{j}(x)$ and $G_{j}^{D}\left(x / x_{1}\right)$ can be found in [13,26-29].

The distribution function $\varphi_{n}^{h}(s, x)$ for the case of $\Lambda_{c}$ production in $p p$ collisions can be written $[26,35]$ as follows:

$$
\begin{aligned}
\varphi_{n}^{\Lambda_{c}}(s, x)= & a_{1}^{\Lambda_{c}}\left[F_{1 q q}^{\Lambda_{c}(n)}\left(x_{+}\right)+F_{1 q q}^{\Lambda_{c}(n)}\left(x_{-}\right)\right] \\
& +a_{0}^{\bar{\Lambda}_{c}}\left[F_{q}^{\Lambda_{c}(n)}\left(x_{+}\right) F_{0 q q}^{\Lambda_{c}(n)}\left(x_{-}\right)\right. \\
& +F_{0 q q}^{\Lambda_{c}(n)}\left(x_{+}\right) F_{q}^{\Lambda_{c}(n)}\left(x_{-}\right) \\
& \left.+2(n-1) F_{q_{\mathrm{sea}}}^{\Lambda_{c}(n)}\left(x_{+}\right) F_{\bar{q}_{\mathrm{sea}}}^{\Lambda_{c}(n)}\left(x_{-}\right)\right] .
\end{aligned}
$$

Here $F_{1 q q}$ denotes the distribution at the leading diquark fragmentation with weight $a_{1}^{\Lambda_{c}}$ and $F_{0 q q}$ is the distribution for the nonleading fragmentation of diquarks written with the central density parameter $a_{0}^{\bar{\Lambda}_{c}}$ :

$$
\begin{aligned}
& F_{1 q q}^{\Lambda_{c}(n)}\left(x_{ \pm}\right) \\
& =\frac{2}{3} \int_{x_{ \pm}}^{1} f_{p}^{u d(n)}\left(x_{1}\right) G_{1 u d}^{\Lambda_{c}}\left(x_{ \pm} / x_{1}\right) \mathrm{d} x_{1}
\end{aligned}
$$




$$
\begin{aligned}
& \quad+\frac{1}{3} \int_{x_{ \pm}}^{1} f_{p}^{u u(n)}\left(x_{1}\right) G_{1 u u}^{\Lambda_{c}}\left(x_{ \pm} / x_{1}\right) \mathrm{d} x_{1} \\
& F_{0 q q}^{\Lambda_{c}(n)}\left(x_{ \pm}\right) \\
& =\frac{2}{3} \int_{x_{ \pm}}^{1} f_{p}^{u d(n)}\left(x_{1}\right) G_{0 u d}^{\Lambda_{c}}\left(x_{ \pm} / x_{1}\right) \mathrm{d} x_{1} \\
& \quad+\frac{1}{3} \int_{x_{ \pm}}^{1} f_{p}^{u u(n)}\left(x_{1}\right) G_{0 u u}^{\Lambda_{c}}\left(x_{ \pm} / x_{1}\right) \mathrm{d} x_{1}, \\
& F_{q}^{\Lambda_{c}(n)}\left(x_{ \pm}\right) \\
& =\frac{2}{3} \int_{x_{ \pm}}^{1} f_{p}^{u_{V}(n)}\left(x_{1}\right) G_{u}^{\Lambda_{c}}\left(x_{ \pm} / x_{1}\right) \mathrm{d} x_{1} \\
& \quad+\frac{1}{3} \int_{x_{ \pm}}^{1} f_{p}^{d_{V}(n)}\left(x_{1}\right) G_{d}^{\Lambda_{c}}\left(x_{ \pm} / x_{1}\right) \mathrm{d} x_{1}, \\
& F_{q_{s e a}}^{\Lambda_{c}(n)}\left(x_{ \pm}\right) \\
& =
\end{aligned}
$$

where

$$
\begin{aligned}
& G_{1 u d}^{\Lambda_{c}}\left(x_{ \pm} / x_{1}\right)=\left(x_{ \pm} / x_{1}\right)^{2\left(\alpha_{R}(0)-\alpha_{N}(0)\right)}\left(1-x_{ \pm} / x_{1}\right)^{\chi} \\
& G_{1 u u}^{\Lambda_{c}}\left(x_{ \pm} / x_{1}\right)=\left(x_{ \pm} / x_{1}\right)^{2}\left(1-x_{ \pm} / x_{1}\right)^{\chi+1} \\
& G_{0 u d}^{\Lambda_{c}}\left(x_{ \pm} / x_{1}\right)=G_{0 u u}^{\Lambda_{c}}=\left(1-x_{ \pm} / x_{1}\right)^{\chi+4\left(1-\alpha_{N}(0)\right)} \\
& G_{u}^{\Lambda_{c}}\left(x_{ \pm} / x_{1}\right)=G_{d}^{\Lambda_{c}}=\left(1-x_{ \pm} / x_{1}\right)^{\chi+2\left(\alpha_{R}(0)-\alpha_{N}(0)\right)}, \\
& G_{\bar{u}}^{\Lambda_{c}}\left(x_{ \pm} / x_{1}\right)=G_{\bar{d}}^{\Lambda_{c}} \\
& \quad=\left(1-x_{ \pm} / x_{1}\right)^{\chi+2\left(\alpha_{R}(0)-\alpha_{N}(0)\right)+2\left(1-\alpha_{R}(0)\right)} \\
& \chi=\lambda-\alpha_{\psi}(0)
\end{aligned}
$$

Charmed baryons $\Lambda_{c}$ have a harder spectrum than charmed mesons in the region $x>0.1$. The fragmentation process of the charmed baryon differs from the fragmentation of $D$ mesons since the $\Lambda_{c}$ baryon consists of three quarks. The diquark fragmentation functions are divided into two parts, which describe different kinematical regions: $F_{0 q q}^{n}(x)$ (central region) and $F_{1 q q}^{n}(x)$ (fragmentation region).

The first term in (13) corresponds to direct $\Lambda_{c}^{+}$baryon production and the second one is connected to a pair production of $\Lambda_{c}^{+} / \Lambda_{c}^{-}$. In the $p p$ interactions the $\Lambda_{c}^{+}$baryon can be pro- duced by a leading $u d$ diquark, which leads to enhancement of $\Lambda_{c}^{+}$spectra in comparison with $\Lambda_{c}^{-}$. Accounting for the sea diquark contribution is important only for antibaryon production in the forward region, while for baryon spectra the sea diquarks contribute little practically at all $x$ [36]. At $x \rightarrow 1$ direct $\Lambda_{c}^{+}$production dominates over the pair $\Lambda_{c}^{+} / \Lambda_{c}^{-}$production because the diquark fragmentation function for the pair production is suppressed in the forward region by the additional factor $\left(1-x_{ \pm} / x_{1}\right)^{4\left(1-\alpha_{N}(0)\right)}$. So here we neglect the sea diquark contribution.

The distribution functions of charmed particles in (5) contain free parameters that cannot be calculated within the framework of the quark-gluon string model, and their values should be found from a comparison with experiments. The intercept $\alpha_{\psi}(0)$ of the poorly studied $c \bar{c}$-trajectory noticeably affects the cross sections of $D$ meson production. Two values of $\alpha_{\psi}(0)$ have been used by the QGSM authors [13,31]: $\alpha_{\psi}(0)=-2.2$, obtained from the mass spectrum on the assumption of linear Regge trajectory, and $\alpha_{\psi}(0)=0$ (nonlinear trajectory), derived from the perturbative calculations. Based on the experimental data on $\Lambda_{c}$ production, they consider [31] the nonperturbative value $\alpha_{\psi}(0)=-2.2$ as the preferable one. If the Regge trajectory $\alpha_{\psi}(t)$ is linear (similar to light hadrons), then the $x$-distributions of the charmed particles become softer than the case of $\alpha_{\psi}(0)=0$.

The coefficient $a_{1}$ provides an unified description of the kinematic regions $x \rightarrow 0$ and $x \rightarrow 1$ in the case of leading fragmentation (when the valence quarks take part in the fragmentation). Now there are no clear arguments for the choice of its value, and different authors apply various values among which two extreme values may be chosen: $a_{1}=2$ [30] and $a_{1}=30$ [13]. New measurements of the total cross sections of charmed meson production at high energies in the experiments ALICE [16-18] and ATLAS [19] allow for a check of the QGSM predictions for extreme values of the parameter $a_{1}$.

The parameter $a^{h}$ in (5) concerns the charmed particle number density in the central region of the inclusive spectra and in an obvious way affects the cross sections in (1). For the $D$ and $\Lambda_{c}^{+}$particles, we use the values from Refs. [13,30]: $a^{D}=7.0 \cdot 10^{-4}, a_{0}^{\bar{\Lambda}_{c}}=7 \cdot 10^{-4}, a_{1}^{\Lambda_{c}}=0.12\left(\right.$ for $\alpha_{\psi}(0)=$ -2.2 ) and $a_{1}^{\Lambda_{c}}=0.02$ (for $\alpha_{\psi}(0)=0$ ). The value $a^{D}=$ $1.0 \cdot 10^{-3}$ is also acceptable for $\mathrm{LHCb}$ experimental data.

The results of the calculation of the cross sections of $D$ meson production in $p p$ collisions in comparison with experimental data are shown in Figs. 1, 2, and 3. The total cross section of $D / \bar{D}$ meson production in $p p$ collisions as a function of center-of-mass energy is calculated in the QGSM for four sets of free parameters (Fig. 1). Here the experimental results in a wide energy range [37-45] including LHC measurements [16-19] are presented. Calculation with $\alpha_{\psi}(0)=0$ and $a_{1}=30$ does not agree with 


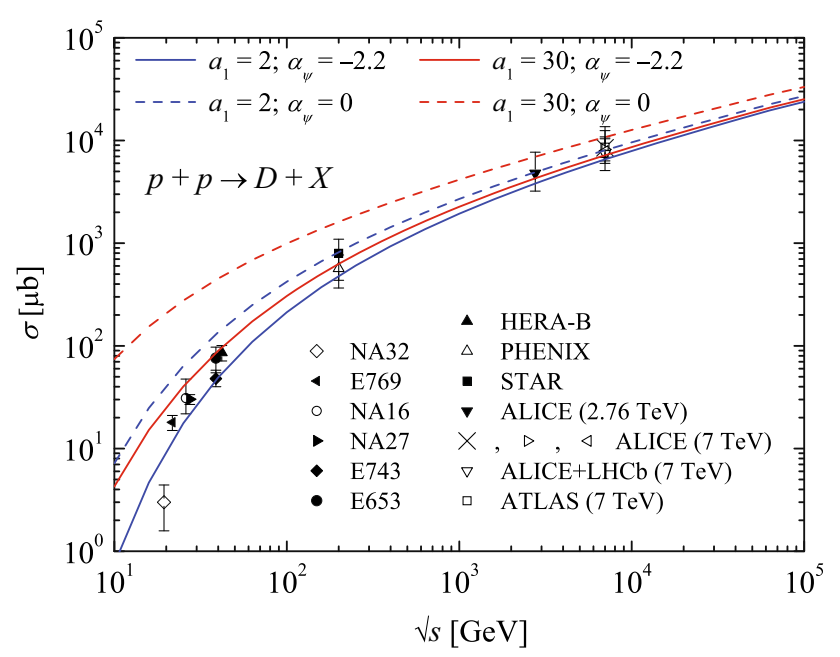

Fig. 1 Total cross sections of $D / \bar{D}$ mesons production in $p p$ collisions. QGSM calculations: $\alpha_{\psi}(0)=-2.2$ (solid lines), $\alpha_{\psi}(0)=0$ (dashed lines); blue (bottom): $a_{1}=2$; red: $a_{1}=30$. The experimental data are taken from Refs. [16-19,37-45]

experimental data at $\sqrt{s}<1 \mathrm{TeV}$, while the calculations with $\alpha_{\psi}(0)=-2.2$ are in close agreement with the measurements in a wide energy range. At low energies the cross sections calculated with $\alpha_{\psi}(0)=-2.2$ for the extreme values $a_{1}=2$ and $a_{1}=30$ differ by a factor $2-5$, but the influence of the parameter $a_{1}$ tends to diminish with energy and becomes negligible at high energies $(\sqrt{s}>1 \mathrm{TeV})$.

Figure 2 represents the calculated differential cross sections of $D$ mesons production at the laboratory energies 400 $\mathrm{GeV}$ and $800 \mathrm{GeV}$ in comparison with the measurements of experiments NA27 [40] and E743 [41].

As can be seen from Figs. 2, 3, QGSM with the intercept $\alpha_{\psi}(0)=-2.2$ better describes the experimental data for differential cross sections. Figure 3 presents the comparison of the experimental data ( $p p$ collisions, $400 \mathrm{GeV}$ ) [40] with calculations of differential cross sections for each sort of $D$ mesons production $\left(D^{+}, D^{-}, D^{0}, \bar{D}^{0}\right)$. The cross sections of $D^{+}$and $D^{0}$ weakly depend on the parameter $a_{1}$, while cross sections of $D^{-}$and $\bar{D}^{0}$ mesons calculated for $a_{1}=2$ are smaller (e.g. for $\alpha_{\psi}(0)=-2.2$ by a factor $2-10$ ) as compared to the case of $a_{1}=30$. The intercept $\alpha_{\psi}(0)=$ -2.2 noticeably better describes the experimental data for $D^{-}$and $\bar{D}^{0}$, whereas the values $\alpha_{\psi}(0)=0, a_{1}=2$ lead to a better description of the $D^{0}$ mesons. In spite of the data spread for $D^{+}$, the intercept $\alpha_{\psi}(0)=-2.2$ seems preferable.

The factor $a_{1}$ amplifies the contribution of the leading fragmentation $\left(D^{-} / \bar{D}^{0}\right)$ with participation of the valence quarks. Production of $D^{-}$and $\bar{D}^{0}$ in $p p$ interactions has a higher probability because these mesons contain the valence quarks of colliding protons. The contribution of the leading fragmentation functions dominates, and $x$-distribution of $D^{-}$ and $\bar{D}^{0}$ is hard in comparison with $D^{+}$and $D^{0}$. The influence of the parameter $a_{1}$ on the cross section of all $D$ mesons production is also noticeable (Fig. 2).

The calculations of cross sections of charmed meson production in $\pi^{-} p$ collisions are compared to experimental data in Figs. 4, 5, 6, and 7. Figure 4 shows the differential cross sections of neutral $D^{0} / \bar{D}^{0}$ mesons production in $\pi^{-} p$ collisions at energy $500 \mathrm{GeV}$ in comparison to the data of the experiment E791 [46]. The calculation for the values $\alpha_{\psi}(0)=-2.2$ and $a_{1}=2$ agrees with the measurement data in the small $x$ range, and a prediction obtained for $\alpha_{\psi}(0)=-2.2$ and $a_{1}=30$ better describes the experimental data in the fragmentation region. The differential cross sections of $D / \bar{D}$ mesons computed at energy $360 \mathrm{GeV}$ in comparison to the data of experiments WA92 $(350 \mathrm{GeV})$ [47] and NA27 (360 GeV) [48] are presented in Fig. 5. In spite of small differences of the beam energies, data of the
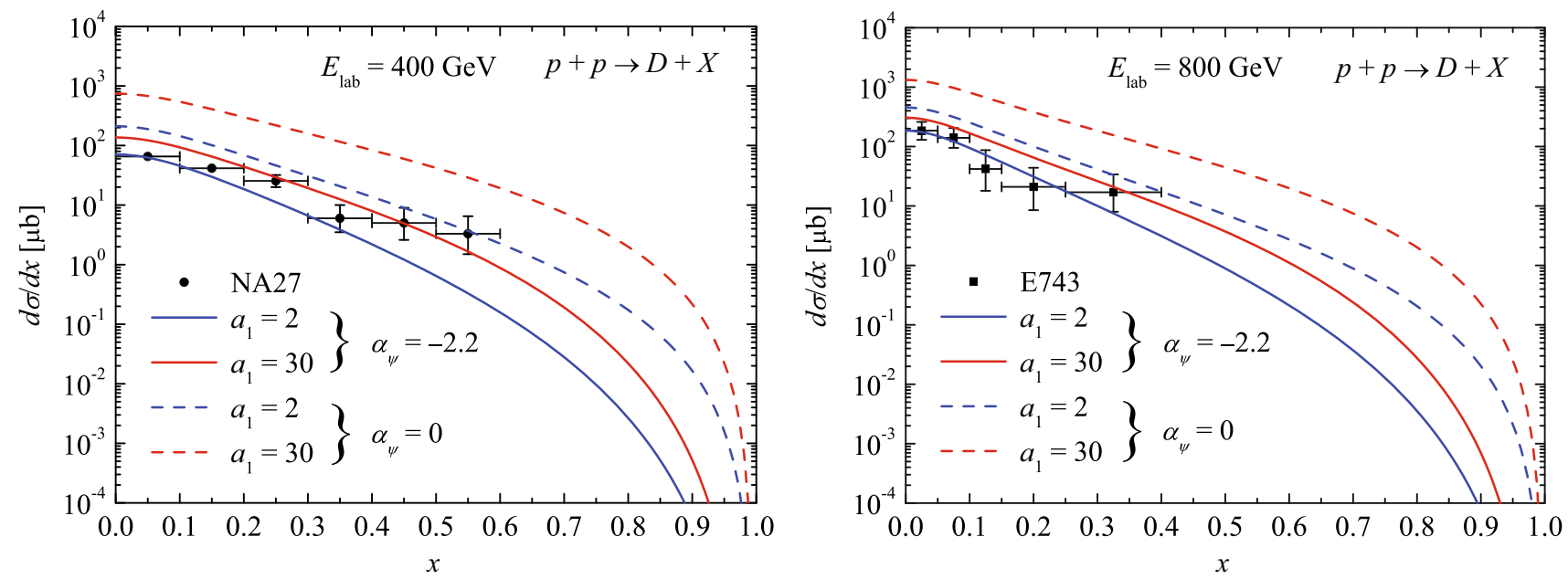

Fig. 2 Differential cross sections of $D / \bar{D}$ mesons production in $p p$ collisions at $E_{\text {lab }}=400 \mathrm{GeV}$ (left) and $E_{\text {lab }}=800 \mathrm{GeV}$ (right). Experimental data: closed circle for [40]; closed square for [41]. Same notation for lines as in Fig. 1 

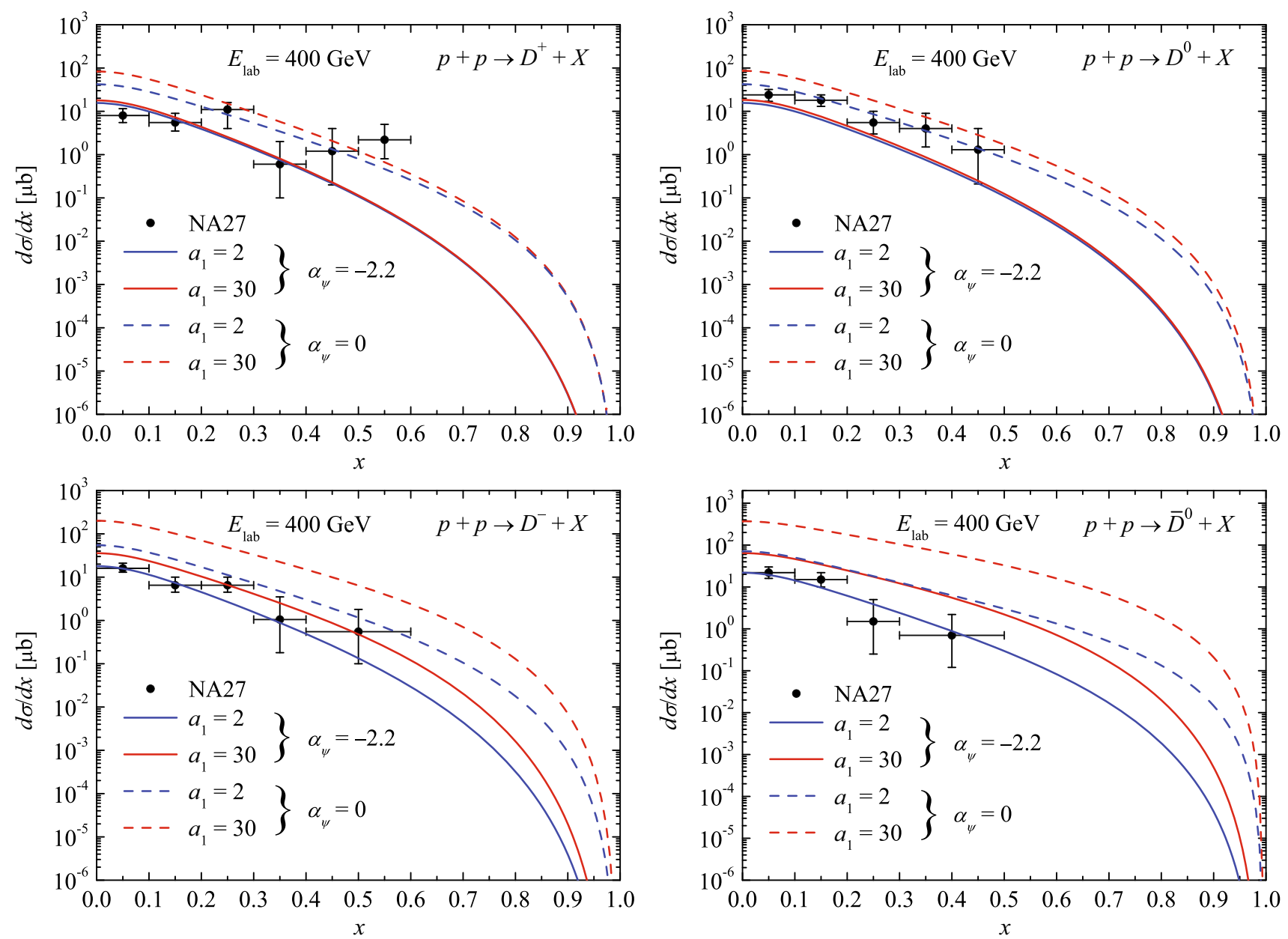

Fig. 3 Differential cross sections of each type of $D$ mesons $\left(D^{+}, D^{-}, D^{0}, \bar{D}^{0}\right)$ production in $p p$ collisions at $E_{\text {lab }}=400 \mathrm{GeV}$. Lines plot the result of QGSM calculations with $\alpha_{\psi}(0)=-2.2$ (solid lines) and $\alpha_{\psi}(0)=0$ (dash). Experimental data are from [40]

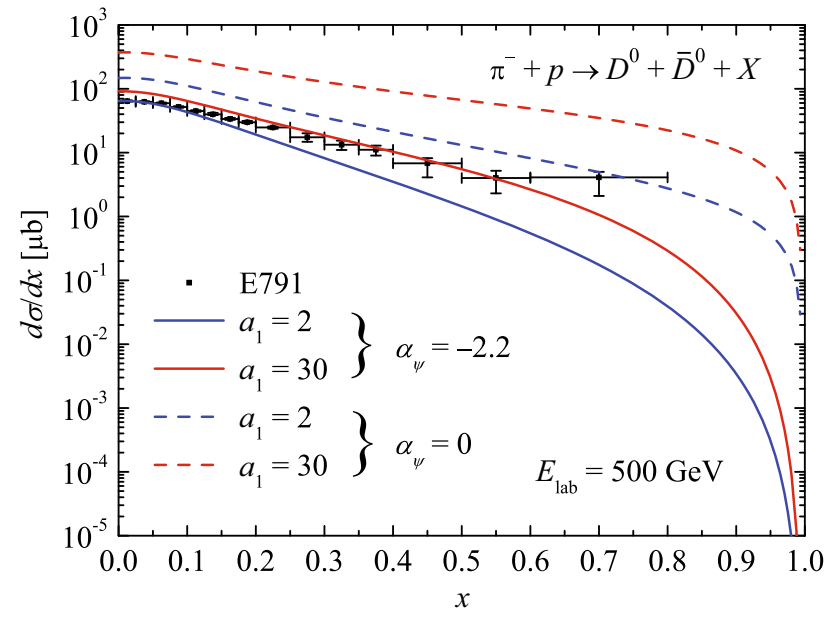

Fig. 4 Differential cross sections of $D^{0} / \bar{D}^{0}$ mesons production in $\pi p$ collisions at $E_{\text {lab }}=500 \mathrm{GeV}$. Experimental data are from [46]. Same notation for lines as in Fig. 3

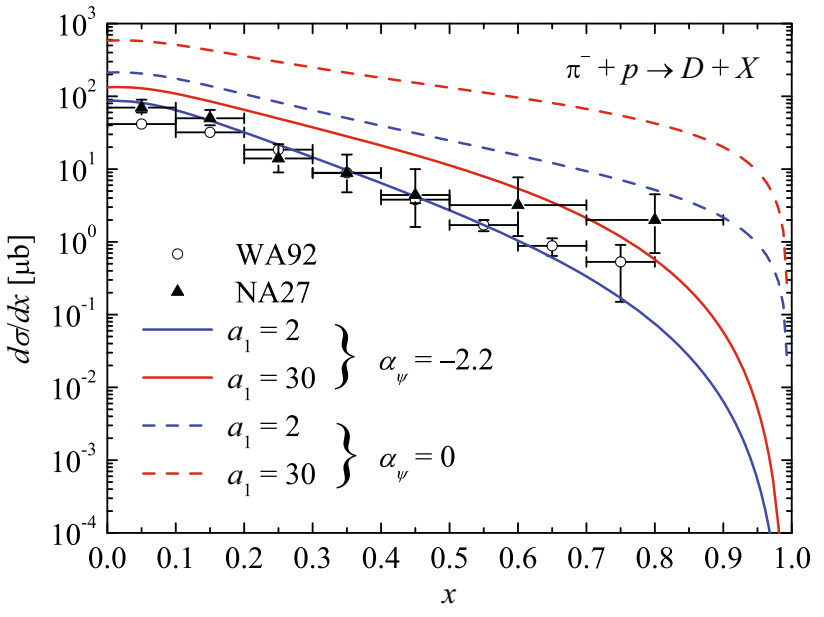

Fig. 5 Differential cross sections of $D / \bar{D}$ mesons production in $\pi p$ collisions calculated in the QGSM at $E_{\text {lab }}=360 \mathrm{GeV}$. Same notation for lines as in Fig. 3. Experimental data for $E_{\text {lab }}=350 \mathrm{GeV}$ (WA92) [47], and for $E_{\mathrm{lab}}=360 \mathrm{GeV}$ (NA27) [48] 

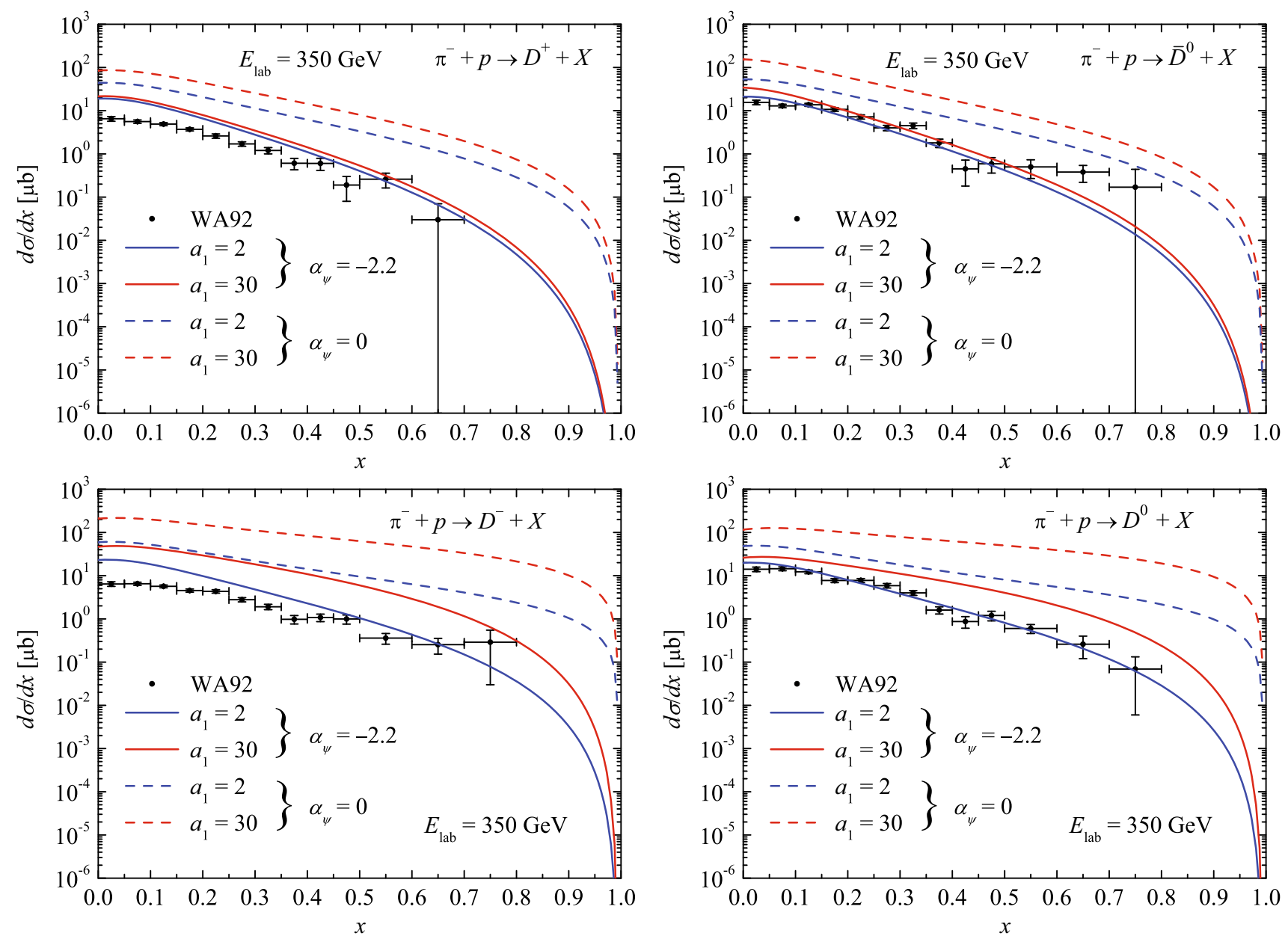

Fig. 6 Differential cross sections of each type of $D$ mesons $\left(D^{+}, D^{-}, D^{0}, \bar{D}^{0}\right)$ production in $\pi p$ collisions at $E_{\text {lab }}=350 \mathrm{GeV}$. QGSM calculations for $\alpha_{\psi}(0)=-2.2$ (solid lines) and $\alpha_{\psi}(0)=0$ (dash). Points are experimental data from [47]

two experiments differ at small $x$ and at $x>0.5$. The calculation for $\alpha_{\psi}(0)=-2.2$ and $a_{1}=2$ describes well most of experimental points.

The differential cross sections for each type of $D$ mesons in comparison to measurements (WA92) are shown in Fig. 6. Calculations with $\alpha_{\psi}(0)=-2.2$ show good agreement with the data on the neutral $D$ meson production. On the other hand the intercept $\alpha_{\psi}(0)=-2.2$ gives overestimated cross sections in the case of charged $D$ meson production at $x<0.5$. As one can see from Figs. 5, 6, and 7, there is similar dependence on the parameter $a_{1}$ of $D$ meson cross sections in $p p$ collisions (see Figs. 2 and 3): $x$-distributions of leading particles are more sensitive to $a_{1}$. The only difference is that $D^{-}$and $D^{0}$ produced in $\pi^{-} p$ interactions are leading particles unlike $D^{+}$and $\bar{D}^{0}$ by virtue of the different quark composition of the colliding particles.

The calculations of the leading and nonleading differential cross sections of $D$ mesons production in $\pi^{-} p$ collisions at energy $360 \mathrm{GeV}$ (LF) are shown in Fig. 7 along with experimental data of NA27 [48].
A comparison of the cross section of $\Lambda_{c}^{+}$baryon production in $p p$ collisions with experimental data is shown in Fig. 8. The differential cross section was calculated at $\sqrt{s}=62 \mathrm{GeV}$ (left panel); the experimental data were obtained for energies $\sqrt{s}=62 \mathrm{GeV}$ [49] and $63 \mathrm{GeV}$ [50]. There is an appreciable difference of the cross section measurements in these two experiments. The calculation with the parameter $\alpha_{\psi}(0)=-2.2$ agrees with the latter experiment.

The right panel of Fig. 8 shows the total cross section of $\Lambda_{c}^{+}$production as a function of center-of-mass energy. The experimental points are taken from Ref. [49], the calculation was made for the same parameter sets. The large spread of the total cross section data prevents from making a definite choice of the intercept $\alpha_{\psi}(0)$.

In Figs. 9, 10, and 11, we show the differential cross sections of $D$ mesons production in $p p$ collisions as a function of the rapidity $(y)$ in comparison to LHCb measurements. Experimental data were obtained at energies $\sqrt{s}=5$ $\mathrm{TeV}$ [51], $7 \mathrm{TeV}$ [52] and $13 \mathrm{TeV}$ [53] for the rapidity range $2 \leqslant y \leqslant 4.5$, which corresponds to $x \lesssim 10^{-3}-10^{-2}$. The 

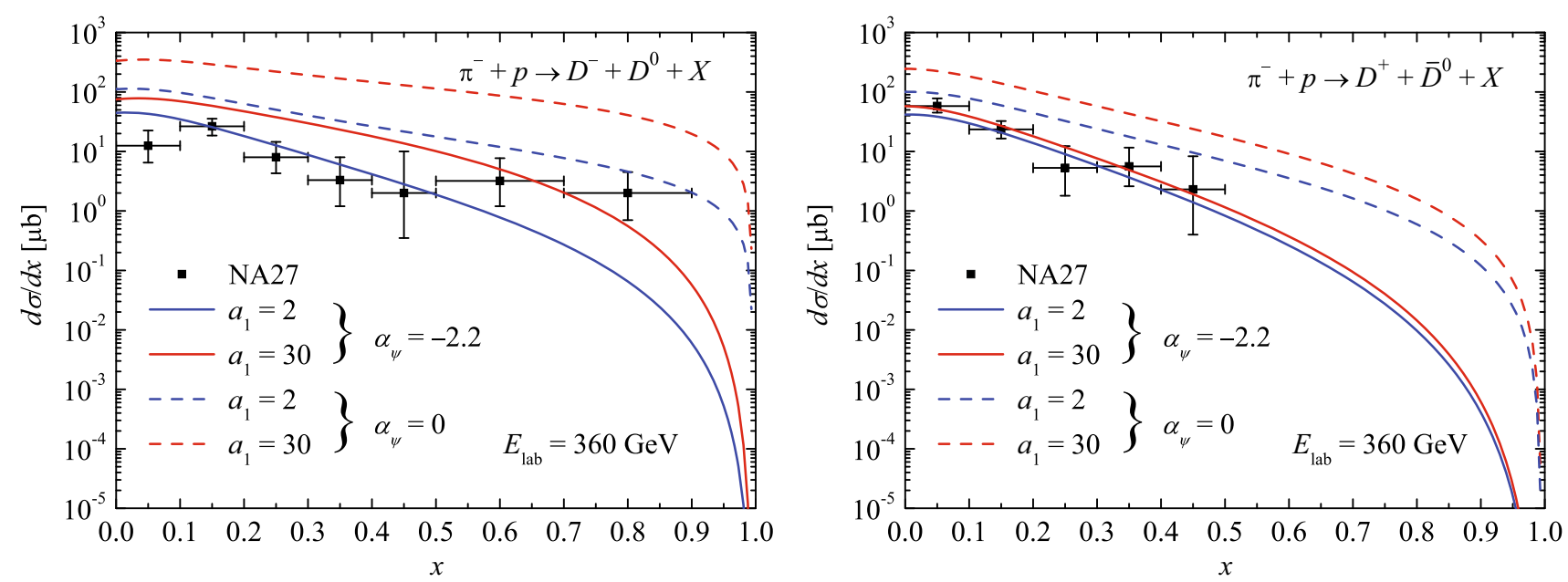

Fig. 7 Differential cross sections of "leading" $D^{-} / D^{0}$ mesons (left) and "nonleading" $D^{+} / \bar{D}^{0}$ mesons (right) in $\pi p$ collisions at $E_{\text {lab }}=360 \mathrm{GeV}$. Calculations for $\alpha_{\psi}(0)=-2.2$ (solid line) and $\alpha_{\psi}(0)=0$ (dash). Points are the data of experiment [48]
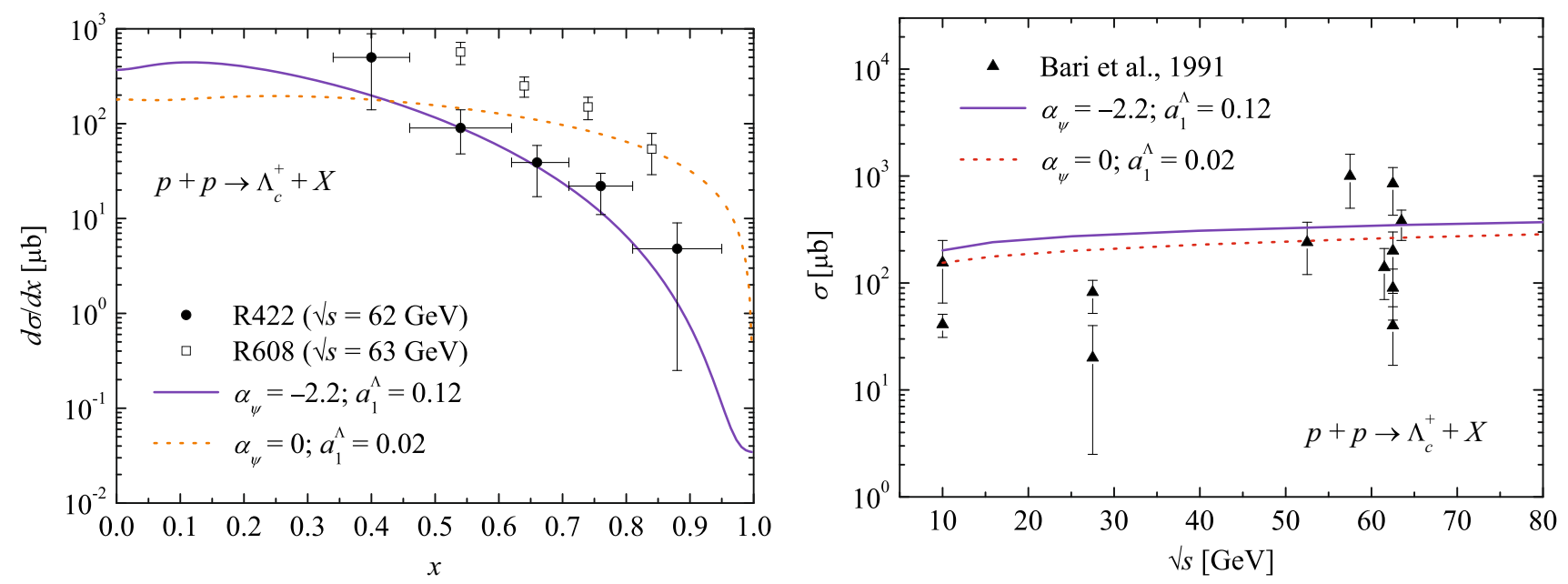

Fig. 8 Differential (left) and total (right) cross sections of $\Lambda_{c}^{+}$baryon production in $p p$ collisions: calculations for $\alpha_{\psi}(0)=-2.2, a_{1}^{\Lambda_{c}}=0.12$ ( line) and $\alpha_{\psi}(0)=0, a_{1}^{\Lambda_{c}}=0.02$ (dotted line). Experimental data: filled circle, filled triangle for [49]; unfilled square for [50]

points plotted in Figs. 9, 10, and 11 were obtained from the original experimental data by summing them over the transverse momentum bins (for each bin in $y$ ).

The problem is expected of describing the experimental data on $D$ mesons in the small $x$ range. In the QGSM version under consideration, the inclusive spectra of charmed particles production are averaged over transverse momentum, while the $\mathrm{LHCb}$ data were obtained for the transverse momentum interval $p_{\perp} \leqslant 15 \mathrm{GeV} / \mathrm{c}$ at the rapidity values 2.0-4.5. However, the calculation with $\alpha_{\psi}(0)=-2.2$ satisfactorily describes the experimental measurements on the production $D^{ \pm}$mesons (unlike $D^{0} / \bar{D}^{0}$ ) at energies $\sqrt{s}=5$ $\mathrm{TeV}$ and $7 \mathrm{TeV}$ (Figs. 9, 10). The experimental data on neutral $D^{0} / \bar{D}^{0}$ and charged $D^{+} / D^{-}$mesons differ by a factor 2-3 (at fixed energy), while QGSM predicts close values of $d \sigma / d y$. That is, the model describes the cross sections of charged mesons much better than $D^{0} / \bar{D}^{0}$. One possible explanation of this discrepancy is that $D^{0} / \bar{D}^{0}$ events contain a mixture of vector mesons $D^{* 0} / \bar{D}^{* 0}$, decays of which might contribute to pseudoscalar $D^{0}$ mesons.

It is possible also that in the case of the small- $x$ events and large transverse momentum at high energy we encounter the problem of "enhanced" diagrams, relating to interactions between pomerons, which are neglected in the quasieikonal approximation. Taking account of enhanced diagrams leads to $x$-distributions, rising as $1 / x$ at small $x$ [31]. Perhaps there will be required a significant revision of the QGSM parameters and calculation technique for this region of kinematics.

Note, however, that the small- $x$ region $\left(10^{-4}-10^{-3}\right)$ gives a minor contribution to the atmospheric neutrino flux because of the dominating peripheric processes in the cosmic-ray induced hadronic cascade: the small $x$ values are suppressed 

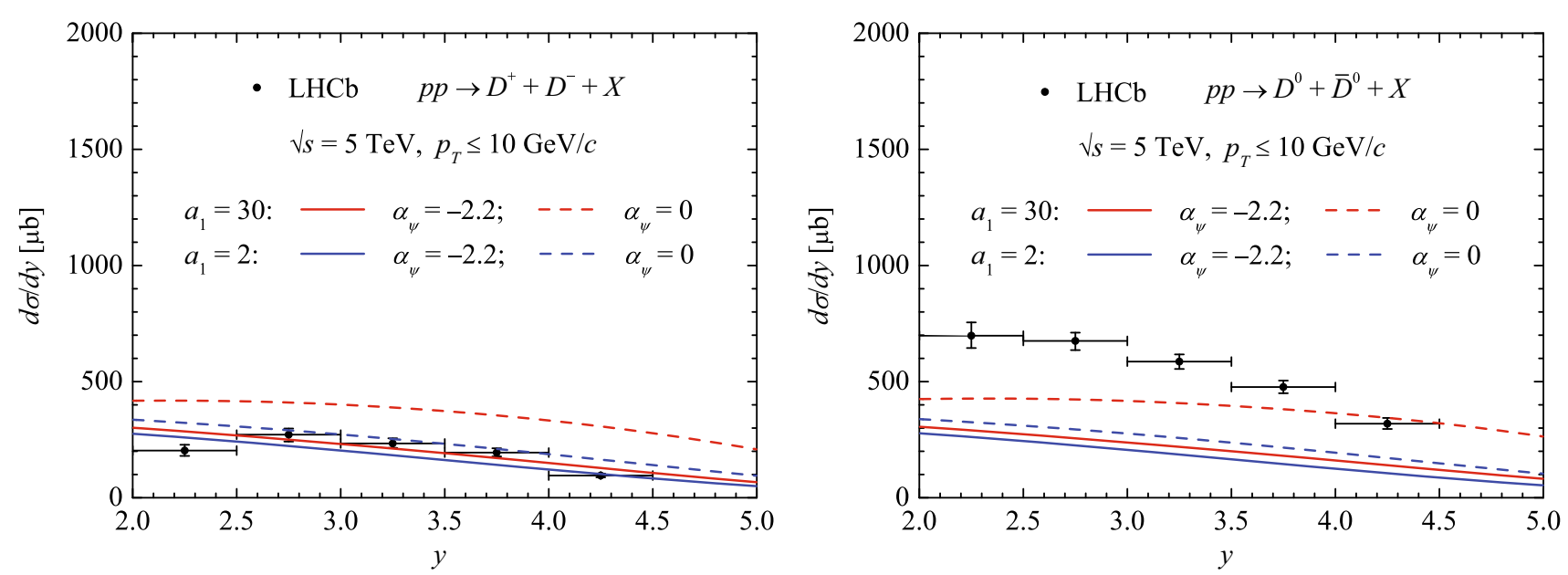

Fig. 9 Differential cross sections of charged $D$ mesons (left) and neutral $D$ mesons (right) in $p p$ collisions at $\sqrt{s}=5 \mathrm{TeV}$. Data are from the LHCb experiment [51]. QGSM calculations: $\alpha_{\psi}(0)=-2.2$ (solid lines), $\alpha_{\psi}(0)=0$ (dash lines); $a_{1}=2$ (blue bottom), $a_{1}=30$ (red)
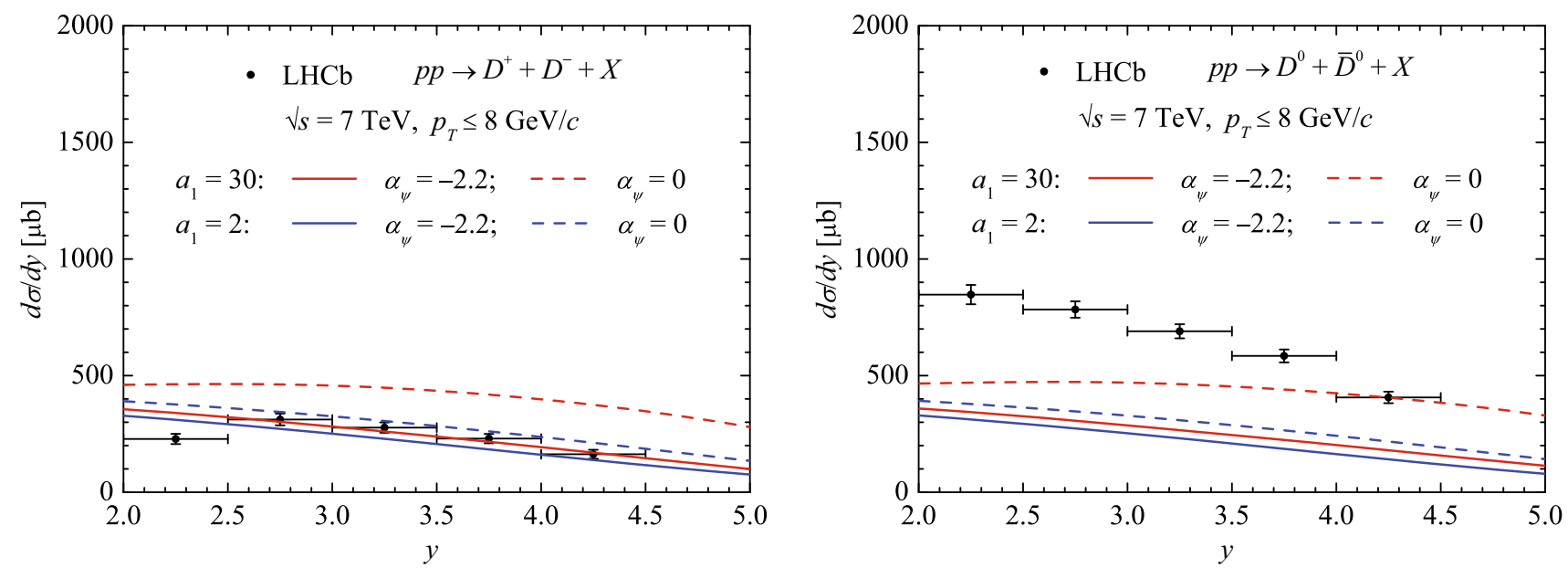

Fig. 10 Differential cross sections of charged $D$ mesons (left) and neutral $D$ mesons (right) in $p p$ collisions at $\sqrt{s}=7 \mathrm{TeV}$. Data are from the LHCb experiment [52]. The same notation for lines as in Fig. 9
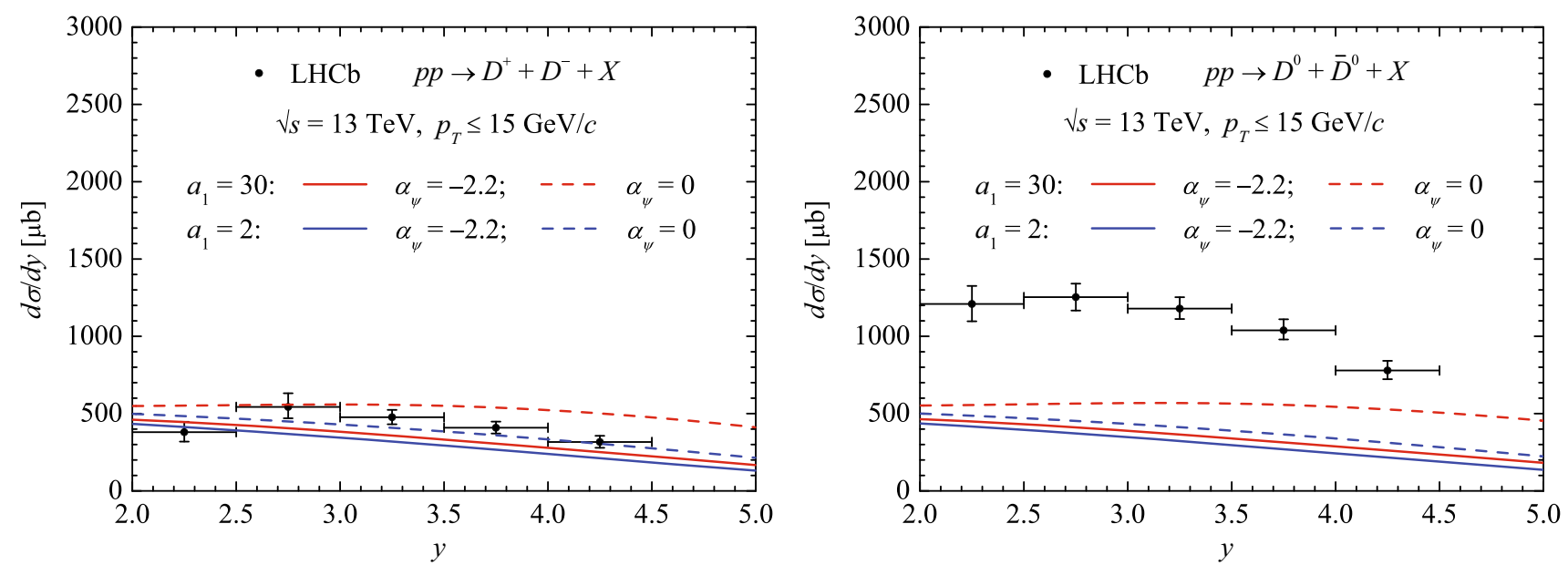

Fig. 11 Differential cross sections of charged $D$ mesons (left) and neutral $D$ mesons (right) in $p p$ collisions at $\sqrt{s}=13 \mathrm{TeV}$. Data are from the LHCb experiment [53]. The same notation for lines as in Fig. 9 

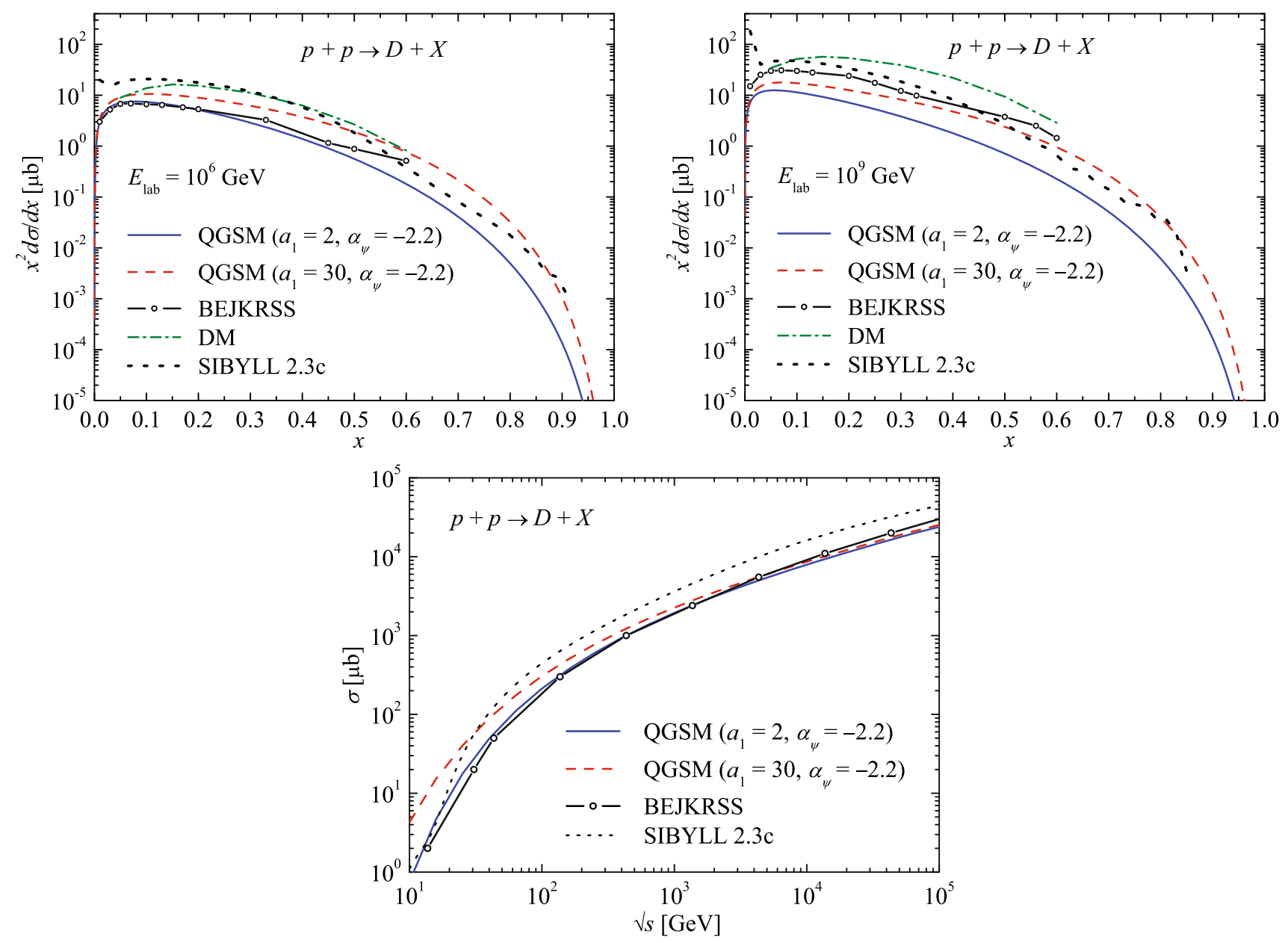

Fig. 12 Model predictions for the differential (top) and total (bottom) cross sections of $D / \bar{D}$ mesons production in $p p$ collisions: QGSM pQCD (BEJKRSS) [24] (line with circles), dipole model (DM) [22] with $\alpha_{\psi}(0)=-2.2$ for $a_{1}=2$ (solid line) and $a_{1}=30$ (dash), NLO
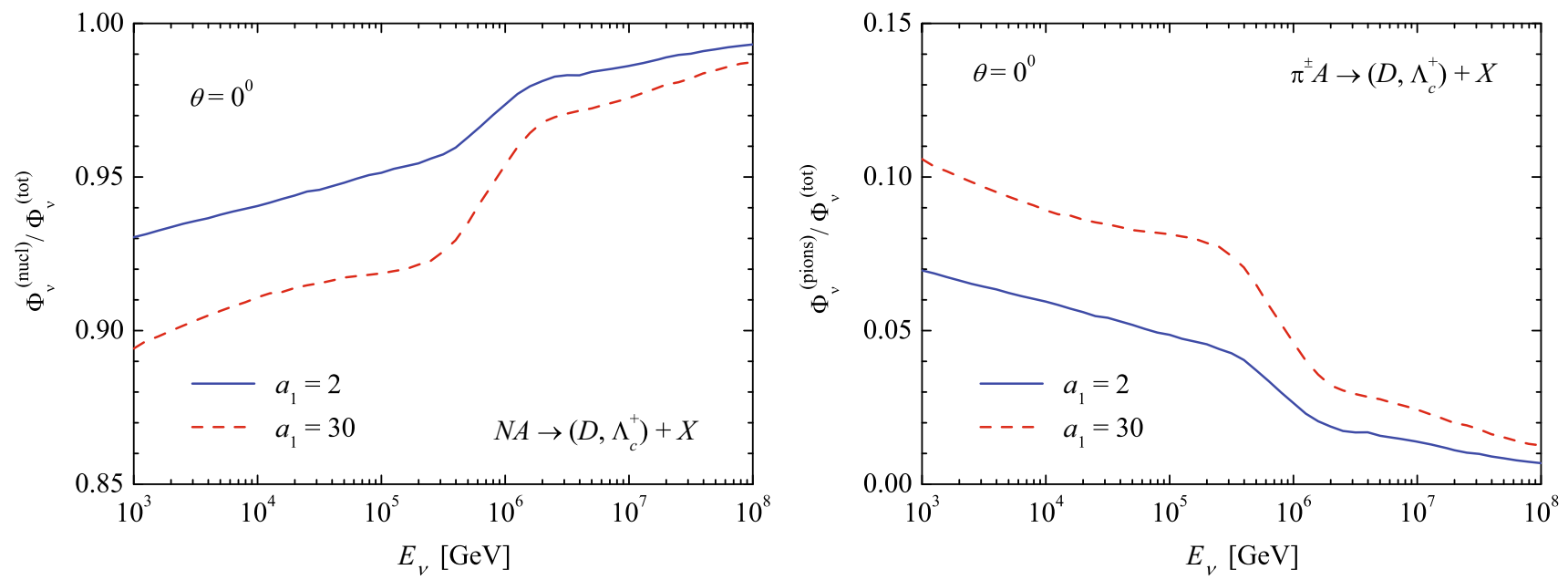

Fig. 13 Partial contributions of $N A$ (left) and $\pi A$ collisions (right) to the prompt muon neutrinos calculated for NSU spectrum with $\alpha_{\psi}(0)=-2.2$ for $a_{1}=2$ (solid line) and $a_{1}=30$ (dash) 
under the integral by a factor $x^{\gamma}$, where $\gamma$ is the spectral index of cosmic-ray protons $(\gamma \approx 1.7-2.0)$.

\section{QGSM in comparison with different charm production models}

Before comparing the prompt atmospheric neutrino fluxes predictions it would be useful to confront the cross sections of the charmed particle production of the different models. The comparison of the differential cross sections of charmed meson production in $p p$ collisions for proton energies in the laboratory frame $\left(10^{3}\right.$ and $\left.10^{6} \mathrm{TeV}\right)$ is shown in the top panel of Fig. 12: QGSM (solid and dashed lines), SIB YLL 2.3c [23] (dotted line), perturbative QCD model (BEJKRSS) [24] (the line with symbols), and the dipole model (DM) [22] (dashdotted). The pQCD calculation is rather close to the present work results obtained with the parameters $\alpha_{\psi}(0)=-2.2$, $a_{1}=2\left(10^{3} \mathrm{TeV}\right)$ and $a_{1}=30\left(10^{6} \mathrm{TeV}\right)$. Our calculation lies below the DM result for most of the $x$ range, which should lead to a low prompt neutrino flux as compared with the result of Enberg et al. [22].

The total cross sections of $D$ meson production in $p p$ collisions as a function of the center-of-mass energy are shown in the bottom panel of Fig. 12 for QGSM, SIBYLL 2.3c [23] and pQCD model (BEJKRSS) [24]. Predictions of the QGSM (for $a_{1}=2$ ) and pQCD model are almost the same in a wide energy range, with the exception of energies $\sqrt{s}<100$ $\mathrm{GeV}$ (calculation for $a_{1}=30$ gives large cross sections at $\left.\sqrt{s}<10^{3} \mathrm{GeV}\right)$.

To calculate the prompt neutrino flux one needs to know the cross sections of charmed particles production in collisions of hadrons with atmospheric nuclei. The cross sections are recalculated for a nuclear target with average mass number $A$ according to the formula $d \sigma_{p A} / d x=A^{\alpha} d \sigma_{p p} / d x$ (for the air we take $A=14.5$ ). The index $\alpha$ depends on $x$ : $\alpha \approx 1$ at $x \rightarrow 0$ and monotonically decreases with rise of $x$ $(\alpha \approx 0.5$ at $x \rightarrow 1$ ) [13]. In [15] the prompt neutrino flux has been calculated for constant $\alpha \approx 0.72$ (the authors argued that the uncertainty due to this choice does not exceed $15 \%$ ), and we use the same value.

\section{Energy spectra of the prompt atmospheric neutrinos}

In the present work, the calculation of prompt neutrino fluxes is performed with the method of $[15,20]$ for QGSM for the parameterization of the cosmic-ray spectrum by Nikolsky, Stamenov, Ushev (NSU) [54], the toy model by Thunman, Ingelman, Gondolo (TIG) [55], and the recent model for cosmic-ray spectrum by Hillas and Gaisser (H3a) [56]. The NSU spectrum, which takes into account an elemental composition of primary cosmic rays, was chosen in order to com- pare new result with the old one $[15,21]$. We use also the toy spectrum TIG (elsewhere called the broken power law, BPL) only in order to compare the results of different calculations, including the dipole model prediction [22] which was used by IceCube as a benchmark model. The three-component model with mixed extragalactic population, $\mathrm{H} 3 \mathrm{a}$ was chosen to compare the QGSM calculation with SIBYLL 2.3c [23], the NLO pQCD predictions BEJKRSS [24] and GRRST [25], and with the IceCube experiment limitation on the prompt neutrino flux [1].

Nucleon-nuclear interactions give the main contribution to the prompt neutrino flux, while $\pi^{ \pm} A$ reactions add less than $5-10 \%$ to the prompt neutrino flux in the energy range $10-10^{5} \mathrm{TeV}$. Figure 13 shows the relative contributions of the $N A$ and $\pi A$ interactions to the prompt fluxes calculated for NSU spectrum and two values of the free parameter of the quark-gluon string model: $a_{1}=2$ (solid line) and $a_{1}=30$ (dashed one). Contributions of $D$ mesons and $\Lambda_{c}^{+}$baryons are presented in Fig. 14.

Figure 15 shows the calculation of the prompt atmospheric neutrinos flux (scaled by $E_{v}^{2}$ ): the band represents this work calculation for the NSU spectrum and the QGSM with the intercept $\alpha_{\psi}(0)=-2.2$. The band shows uncertainty due to a change of the parameter $a_{1}$ which ensures a unified behavior of the leading fragmentation functions at $x \rightarrow 0$ and $x \rightarrow 1$. Extreme values of $a_{1}$ lead to a change of the neutrino flux by a factor 1.4: $a_{1}=2$ corresponds to the lower bound, and $a_{1}=30$ to the upper one. However, the influence of the intercept of the Regge trajectory $\alpha_{\psi}(0)$ appears to be more substantial: the replacement of $\alpha_{\psi}(0)=0$ by $\alpha_{\psi}(0)=-2.2$ reduces the flux by a factor 3 as compared to the result [15] (solid line) obtained for the same scheme (QGSM+NSU) with intercept $\alpha_{\psi}(0)=0$. The dipole model result [22] for the TIG cosmic-ray spectrum is also shown in Fig. 15 (dashed line).

The QGSM flux [15] performed for $\alpha_{\psi}(0)=0$ and the NSU spectrum was considered by IceCube collaborators as too optimistic prediction $[57,58]$. At the energies $E_{v}>10^{6} \mathrm{GeV}$ it exceeds the ERS result [22] by about $30 \%$; however, part of this excess is related to the difference of the cosmic-ray spectra used.

The influence of charm production models on the neutrino fluxes is seen in Fig. 16. All results are obtained for the same cosmic-ray spectrum TIG. In the energy range beyond $1 \mathrm{PeV}$, where atmospheric neutrinos from charmed particles dominate, QGSM (shaded band) leads to an appreciably lower flux than the dipole model result [22] (dashed line). The predictions of the pQCD models, BEJKRSS [24] and GRRST [25] are compatible in the whole energy range. The QGSM flux at energies above $200 \mathrm{TeV}$ is close to upper bound of the BEJKRSS band.

The uncertainty of the QGSM neutrino flux prediction due to variation of the intercept $\alpha_{\psi}(0)$ is shown in Fig. 17. 

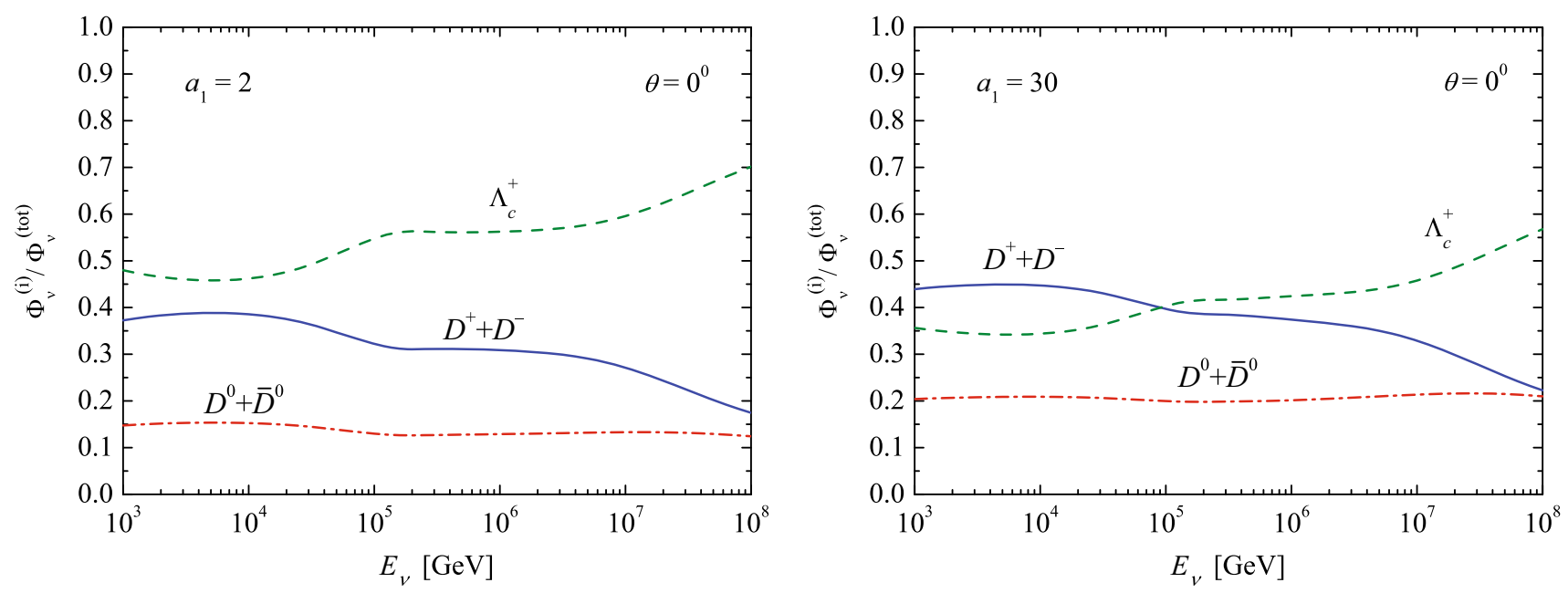

Fig. 14 Partial contributions to the prompt muon neutrino flux calculated with QGSM for NSU spectrum with $\alpha_{\psi}(0)=-2.2$ for $a_{1}=2$ (left) and $a_{1}=30$ (right)

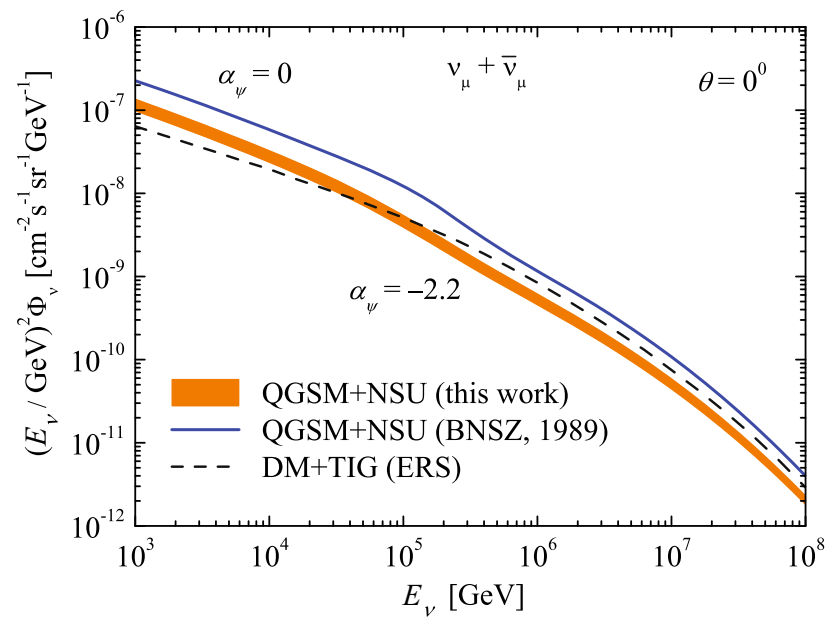

Fig. 15 Vertical flux of prompt atmospheric neutrinos calculated with QGSM for the NSU cosmic-ray spectrum. Shaded band: this work with $\alpha_{\psi}(0)=-2.2$. The band width corresponds to the variation of $a_{1}$ from 2 (lower bound) to 30 (upper bound). The solid line presents the result from Refs. [15,21] obtained with $\alpha_{\psi}(0)=0$. Dash line plots the dipole model calculation [22] for the TIG spectrum

The uncertainty band is computed for $\alpha_{\psi}(0)=-2.2$ with $a^{D}=7.0 \cdot 10^{-4}$ (lower bound) and $\alpha_{\psi}(0)=0$ with $a^{D}=10^{-3}$ (upper bound). The band calculation is performed for H3a cosmic-ray spectrum with use the value $a_{1}=2$. The bulk of prompt neutrino flux uncertainties is induced by intercept of the charm Regge trajectory (as Fig. 17 shows). Rest parameters, $a^{D}, a_{1}$ slightly disturb bounds of the uncertainty span. In fact, the uncertainty due to the $\alpha_{\psi}(0)$ variation absorbs that for the $a_{1}$-variation.

$\alpha_{\psi}(0)=-2.2$ seems to be preferable from the viewpoint of the most experimental measurements on charm production. However, $\alpha_{\psi}(0)=-2.2$ leads to underestimation of the neutral $D$ meson production at high energy. On the other hand

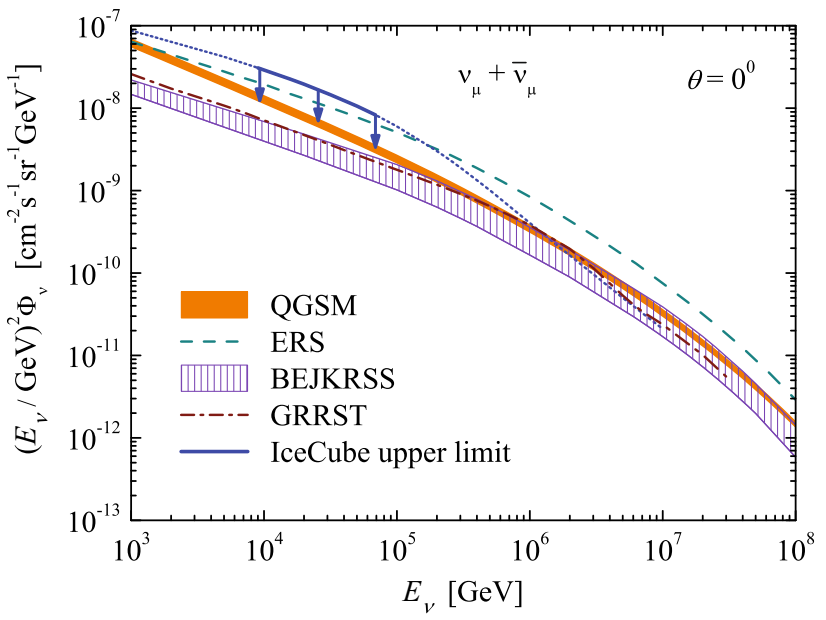

Fig. 16 Comparison of the prompt neutrino flux calculated with use of the models: QGSM with $\alpha_{\psi}(0)=-2.2$ (shaded band), the color dipole model (ERS) [22] (dash line), NLO pQCD models, BEJKRSS [24] (hatched area) and GRRST [25] (dash-dotted line). All calculations are performed for the TIG cosmic-ray spectrum. Solid line denotes the IceCube upper limit and the dotted one its extrapolation [1] (see text for more details)

$\alpha_{\psi}(0)=0$ predicts harder charm spectra at all $x$. The largest differences in $d \sigma / d x$ appear at $x \rightarrow 1$, where the charm spectra calculated for $\alpha_{\psi}(0)=0$ are larger by several orders of magnitude in comparison with case of $\alpha_{\psi}(0)=x \sim 1$ is the region of peripheric processes which dominate in the atmospheric hadronic cascade, therefore atmospheric neutrino fluxes are sensitive to the intercept value: the replacement of $\alpha_{\psi}(0)=-2.2$ by $\alpha_{\psi}(0)=0$ increases the flux by a factor $2-3$.

Relative to the BEJKRSS result, the QGSM gives a large flux at low and middle energies. However, our calculation comes close to the BEJKRSS one with increasing energy, 


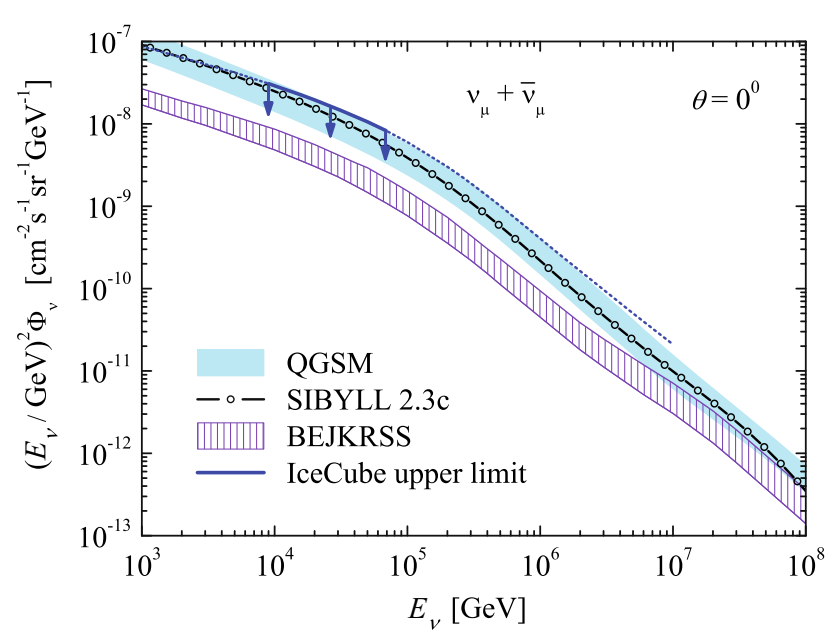

Fig. 17 Uncertainties of the prompt neutrino flux calculations. The blue band corresponding to QGSM calculations shows the effect of varying crucial parameter: the intercept $\alpha_{\psi}(0)=0$ (upper bound of the band) and $\alpha_{\psi}(0)=-2.2$ (lower bound). The hatched area presents the scale of uncertainties of the flux calculation with the NLO pQCD model BEJKRSS [24]. The SIBYLL 2.3c prediction [23] is compatible with QGSM (the line inside band). All calculations are performed for the H3a cosmic-ray spectrum. The solid line with arrows represents the IceCube limitation and dotted line labels its extrapolation [1]

and the QGSM lower bound is compatible with upper bound of the pQCD prediction at $E_{v}>10 \mathrm{PeV}$. On the other hand, our uncertainty band completely covers the SIBYLL 2.3c flux in the whole energy range.

Figure 18 presents our preferred result: the prompt neutrino spectrum calculated with QGSM for the magnitudes of parameters $\alpha_{\psi}(0)=-2.2, a^{D}=7 \cdot 10^{-4}$. The brown band marks the QGSM uncertainty related to the parameter $a_{1}$, which rises from 2 (lower bound) to 30 (upper bound). Also shown here are the SIBYLL 2.3c [23] calculation and the NLO pQCD ones (BEJKRSS [24] and GRRST [25] models). All these spectra were obtained for the H3a cosmic-ray spectrum. The QGSM flux for $a_{1}=30$ and $\alpha_{\psi}(0)=-2.2$ is close to the SIBYLL 2.3c calculation beyond $100 \mathrm{TeV}$ and greater than the BEJKRSS flux by a factor $\sim 2-4$ at $10^{5}-10^{6} \mathrm{GeV}$. With the energy increase these differences decrease, and our prediction at $E_{v} \gtrsim 10 \mathrm{PeV}$ is close to the BEJKRSS flux.

In the IceCube analysis [1], the upper limit on the prompt atmospheric neutrino flux was obtained using high statistics collected over six years. The prompt neutrino flux was constrained using the color dipole model [22] corrected for the cosmic-ray spectrum parametrization $\mathrm{H} 3 p$ [56]. The solid line with arrows in Figs. 16, 17, and 18 shows the constraint specified by IceCube for the sensitive energy range $9-69$ $\mathrm{TeV}$. The extrapolation beyond $69 \mathrm{TeV}$ was made by IceCube using again the prompt neutrino spectrum [22]: the original result was also brought into accord with the cosmic-ray model H3p [56]. The dotted line in Figs. 17 and 18 just shows

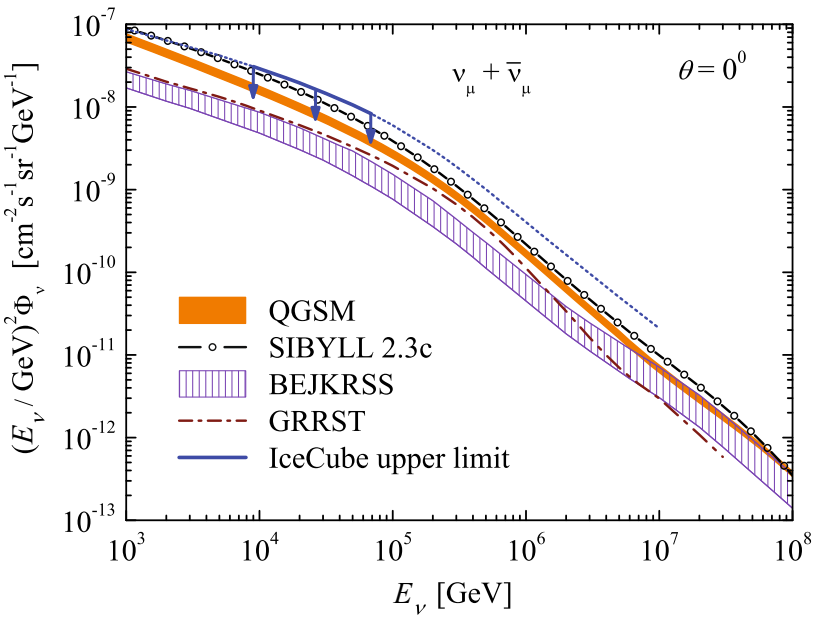

Fig. 18 The comparison of the prompt neutrino flux calculated for the H3a cosmic-ray spectrum [56] with use of different models: QGSM $\left(\alpha_{\psi}(0)=-2.2\right)$, SIBYLL 2.3c [23], the pQCD models BEJKRSS [24] and GRRST [25]. Solid line is the IceCube constraint, dotted line plots the limit extrapolation [1]

the dipole model behavior beyond of the sensitivity range with the best-fit parameters describing the systematic uncertainties of the IceCube analysis, e.g., the optical efficiency of the telescope, the Antarctic ice properties, the uncertainties of the cosmic-ray spectrum.

Our result is evidently compatible with the IceCube limitation, the same is true for the rest of the models under discussion: SIBYLL 2.3c [23], the NLO pQCD models BEJKRSS [24] and GRRST [25].

\section{Conclusion}

The new calculation of the atmospheric neutrino flux from decays of the charmed particles is performed with updated version of the quark-gluon string model. The QGSM parameters $\alpha_{\psi}(0)$ and $a_{1}$ are examined by comparison of calculated cross sections for the charmed meson production with the data of measurements obtained at LHC and in other experiments. Though the data of the $\mathrm{LHCb}$ experiment do not allow a unique choice of the varying parameters magnitudes, we consider the intercept $\alpha_{\psi}(0)=-2.2$ as a preferred value against $\alpha_{\psi}(0)=0$. Note that $\alpha_{\psi}(0)=-2.2$ appears to be the proper magnitude, because it is in accordance also with the observable (and natural) pattern: heavier quarks have a lower intercept of the Regge trajectory.

At high energy the differential cross sections of the charm production are more sensitive (as compared with the total cross section) to the change of the parameter $a_{1}$, which brings the neutrino flux deviation to about $(20-40) \%$ for extreme values of $a_{1}$. The analysis shows that the intercept of the Regge trajectory $\alpha_{\psi}(0)$ causes a more noticeable effect on the 
charm production and therefore on the prompt atmospheric neutrino flux. An updated version of QGSM with $\alpha_{\psi}(0)=$ -2.2 leads to a decrease of the prompt neutrino flux by a factor $\sim 2-3$ as compared to the former QGSM prediction $[15,21]$ obtained with $\alpha_{\psi}(0)=0$.

In the energy range beyond $1 \mathrm{PeV}$, where atmospheric neutrinos from the decay of charmed particles dominate, as expected, the new QGSM flux is significantly lower in comparison with the color dipole model [22]. The QGSM flux obtained for intercept $\alpha_{\psi}(0)=-2.2$ and H3a cosmic-ray spectrum is compatible with the NLO pQCD predictions at $E_{v}>10 \mathrm{PeV}$, and the upper bound of our calculation does not differ practically from the SIBYLL $2.3 \mathrm{c}$ result. The updated QGSM calculation of the prompt atmospheric neutrino flux is consistent with the IceCube limitation.

The performed calculations confirm the viability of QGSM as an appropriate phenomenological model of the high-energy hadronic interactions, which allows one to take account of effects beyond the pQCD. The updated version of QGSM is the suitable approach to provide reasonable prediction of the atmospheric prompt neutrino flux. Undoubtedly, the current version QGSM must be developed taking into account the large-mass diffraction dissociation and resonances production. Also all parameters of the model need a comprehensive revision and the thorough fitting by use of recent and future results of high-energy hadronic experiments.

Acknowledgements We are grateful to A. Fedynitch and J. Talbert for kindly providing us with tables of the prompt neutrino flux calculations. This work is supported by the Russian Federation Ministry of Science and Higher Education (agreement No. 075-15-2019-1631, FZZE-20200017). The work of M.S. is supported by the Grant for JINR young scientists and specialists No. 20-202-09.

Data Availability Statement This manuscript has no associated data or the data will not be deposited. [Authors' comment: All of the data used in this paper are available in references therein. All necessary information to reproduce the QGSM cross sections calculations can be found in the paper and in the references.]

Open Access This article is licensed under a Creative Commons Attribution 4.0 International License, which permits use, sharing, adaptation, distribution and reproduction in any medium or format, as long as you give appropriate credit to the original author(s) and the source, provide a link to the Creative Commons licence, and indicate if changes were made. The images or other third party material in this article are included in the article's Creative Commons licence, unless indicated otherwise in a credit line to the material. If material is not included in the article's Creative Commons licence and your intended use is not permitted by statutory regulation or exceeds the permitted use, you will need to obtain permission directly from the copyright holder. To view a copy of this licence, visit http://creativecomm ons.org/licenses/by/4.0/.

Funded by SCOAP ${ }^{3}$.

\section{References}

1. IceCube Collaboration, M.G. Aartsen et al., Observation and characterization of a cosmic muon neutrino flux from the northern hemisphere using six years of IceCube data. Astrophys. J. 833, 3 (2016). arXiv: 1607.08006

2. IceCube Collaboration, M.G. Aartsen et al., Observation of astrophysical neutrinos in six years of IceCube data. PoS(ICRC2017) (1981). arXiv:1710.01191

3. IceCube Collaboration, M.G. Aartsen et al., Astrophysical neutrinos and cosmic rays observed by IceCube. Adv. Space Res. 62, 2902 (2018). arXiv:1701.03731

4. ANTARES Collaboration, A. Albert et al., All-flavor search for a diffuse flux of cosmic neutrinos with nine years of ANTARES Data. ApJL 853, L7 (2018). arXiv:1711.07212

5. ANTARES Collaboration, S. Adrian-Martinez et al., Measurement of the atmospheric $v_{\mu}$ energy spectrum from $100 \mathrm{GeV}$ to $200 \mathrm{TeV}$ with the ANTARES telescope, Eur. Phys. J. C 73, 2606 (2013). arXiv: 1308.1599

6. Baikal Collaboration, A. D. Avrorin et al., Status of the BaikalGVD Neutrino Telescope. EPJ Web Conf. 207, 01003 (2019)

7. Baikal Collaboration, A. D. Avrorin et al., Search for high-energy neutrinos from GW170817 with the Baikal-GVD neutrino telescope. JETP Lett. 108, 12, 787 (2018). arXiv:1810.10966

8. IceCube Collaboration, M. G. Aartsen et al., Evidence for highenergy extraterrestrial neutrinos at the IceCube detector. Science 342, 1,242,856 (2013)

9. IceCube Collaboration, M. G. Aartsen et al., Observation of highenergy astrophysical neutrinos in three years of IceCube data. Phys. Rev. Lett. 113, 101,101 (2014)

10. IceCube and Fermi-LAT and MAGIC and AGILE and ASAS-SN and HAWC and H.E.S.S. and INTEGRAL and Kanata and Kiso and Kapteyn and Liverpool Telescope and Subaru and Swift NuSTAR and VERITAS and VLA/17B-403 Collaborations, M.G. Aartsen et al., Multimessenger observations of a flaring blazar coincident with high-energy neutrino IceCube-170922A. Science 361, 6398, eaat1378 (2018). arXiv:1807.08816

11. IceCube Collaboration, M.G. Aartsen et al., Neutrino emission from the direction of the blazar TXS $0506+056$ prior to the IceCube-170922A alert. Science 361, 6398, 147 (2018). arXiv: 1807.08794

12. A.B. Kaidalov, K.A. Ter-Martirosyan, Multiple production of hadrons at high-energies in the model of quark-gluon strings. Sov. J. Nucl. Phys. 39, 979 (1984)

13. A.B. Kaidalov, O.I. Piskunova, Production of charmed particles in the quark-gluon string model. Sov. J. Nucl. Phys. 43, 994 (1986)

14. A.B. Kaidalov, Pomeranchuk singularity and high-energy hadronic interactions. Phys. Usp. 46, 1121 (2003)

15. E.V. Bugaev, V.A. Naumov, S.I. Sinegovsky, E.S. Zaslavskaya, Prompt leptons in cosmic rays. Nuovo Cim. C 12, 41 (1989)

16. ALICE Collaboration, B. Abelev et al., Measurement of charm production at central rapidity in proton-proton collisions at $\sqrt{s}=$ 2.76 TeV. JHEP 07, 191 (2012). arXiv: 1205.4007

17. ALICE Collaboration, J. Adam et al., D-meson production in $\mathrm{p}-\mathrm{Pb}$ collisions at $\sqrt{s_{N N}}=5.02 \mathrm{TeV}$ and in pp collisions at $\sqrt{s}=7$ TeV. Phys. Rev. C 94, 054908 (2016). arXiv: 1605.07569

18. ALICE Collaboration, S. Acharya et al., Measurement of D-meson production at mid-rapidity in pp collisions at $\sqrt{s}=7 \mathrm{TeV}$. Eur. Phys. J. C 77, 550 (2017). arXiv:1702.00766

19. ATLAS Collaboration, G. Aad et al., Measurement of $D^{* \pm}, D^{ \pm}$ and $D_{s}^{ \pm}$meson production cross sections in $p p$ collisions at $\sqrt{s}=7$ TeV with the ATLAS detector. Nucl. Phys. B 907, 717 (2016). arXiv:1512.02913 
20. A.N. Vall, V.A. Naumov, S.I. Sinegovsky, Hadronic component of high-energy cosmic rays and growth of the inelastic cross-sections. Sov. J. Nucl. Phys. 44, 806 (1986)

21. V.A. Naumov, T.S. Sinegovskaya, S.I. Sinegovsky, The $K_{l 3}$ form factors and atmospheric neutrino flavor ratio at high energies. Nuovo Cim. A 111, 129 (1998). arXiv:hep-ph/9802410

22. R. Enberg, M.H. Reno, I. Sarcevic, Prompt neutrino fluxes from atmospheric charm. Phys. Rev. D 78, 043005 (2008). arXiv:0806.0418

23. A. Fedynitch, F. Riehn, R. Engel, T.K. Gaisser, T. Stanev, Hadronic interaction model SIBYLL 2.3c and inclusive lepton fluxes. Phys. Rev. D 100, 103018 (2019)

24. A. Bhattacharya, R. Enberg, Y.S. Jeong, C.S. Kim, M.H. Reno, I. Sarcevic, A. Stasto, Prompt atmospheric neutrino fluxes: perturbative QCD models and nuclear effects. JHEP 11, 167 (2016). arXiv: 1607.00193

25. R. Gauld, J. Rojo, L. Rottoli, S. Sarkar, J. Talbert, The prompt atmospheric neutrino flux in the light of LHCb. JHEP 02, 130 (2016)

26. A.B. Kaidalov, O.I. Piskunova, Inclusive spectra of baryons in the Quark-Gluon Strings Model. Z. Phys. C 30, 145 (1986)

27. YuM Shabelski, Secondary particle spectra in $\pi p$ and $p p$ collisions in the Quark-Gluon String model. Sov. J. Nucl. Phys. 44, 117 (1986)

28. G.I. Lykasov, G.G. Arakelyan, M.N. Sergeenko, The quark gluon string model: soft and semihard hadronic processes. Phys. Part. Nucl. 30, 343 (1999)

29. G.H. Arakelyan, P.E. Volkovitsky, Charmed particle production in hadron-hadron collisions. Z. Phys. A 353, 87 (1995)

30. G.H. Arakelyan, Charmed sea contribution to the inclusive hadroproduction of the mesons with open charm in the QuarkGluon String Model. Phys. Atom. Nucl. 61, 1570 (1998). arXiv:hep-ph/9711276

31. A.B. Kaidalov, High-energy hadronic interactions (20 years of the Quark-Gluon Strings Model). Phys. Atom. Nucl. 66, 1994 (2003)

32. K.A. Ter-Martirosyan, On the particle multiplicity distributions at high energy. Phys. Lett. B 44, 377 (1973)

33. J. Bleibel, L.V. Bravina, E.E. Zabrodin, How many of the scaling trends in pp collisions will be violated at $\sqrt{s}_{N N}=14 \mathrm{TeV}$ ? Predictions from Monte Carlo quark-gluon string model. Phys. Rev. D 93, 114012 (2016)

34. A.B. Kaidalov, M.G. Poghosyan, Predictions of the Quark-Gluon String model for pp at LHC. Eur. Phys. J. C 67, 397 (2010). arXiv:0910.2050

35. O.I. Piskounova, Leading-particle effects in the spectra of $\Lambda_{c}$ and $\bar{\Lambda}_{c}$ produced in $\Sigma^{-} p, p p$ and $\pi^{-} p$ interactions. Phys. Atom. Nucl. 66, 307 (2003). arXiv:hep-ph/0202005

36. N. Armesto, E.G. Ferreiro, C. Pajares, Y.M. Shabelski, Fast antibaryon production in pp collisions as a result of string fusion. Z. Phys. C 73, 309 (1997)

37. ACCMOR Collaboration, S. Barlag et al., Production of $D, D^{*}$ and $D_{s}$ mesons in $200 \mathrm{GeV} / \mathrm{c} \pi^{-}, K^{-}$and $p$-Si interactions. Z. Phys. C 39, 451 (1988)

38. Fermilab E769 Collaboration, G.A. Alves et al., Forward cross sections for production of $D^{+}, D^{0}, D_{s}, D^{*+}$ and $\Lambda_{c}$ in $250 \mathrm{GeV}$ $\pi^{ \pm}, K^{ \pm}$and p interactions with nuclei. Phys. Rev. Lett. 77, 2388 (1996)

39. LEBC-EHS Collaboration, M. Aguilar-Benitez et al., D meson branching ratios and hadronic charm production cross sections. Phys. Lett. B 135, 237 (1984)

40. LEBC-EHS Collaboration, M. Aguilar-Benitez et al., Charm hadron properties in $400 \mathrm{GeV} / \mathrm{c}$ pp interactions. Z. Phys. C 40, $321(1988)$

41. LEBC-MPS Collaboration, R. Ammar et al., D-meson production in $800-\mathrm{GeV} / \mathrm{c}$ pp interactions. Phys. Rev. Lett. 61, 2185 (1988)
42. Fermilab E653 Collaboration, K. Kodama et al., Charm meson production in $800 \mathrm{GeV} / \mathrm{c}$ proton-emulsion interactions. Phys. Lett. B 263, 573 (1991)

43. HERA-B Collaboration, A. Zoccoli et al., Charm, beauty and charmonium production at HERA-B. Eur. Phys. J. C 43, 179 (2005)

44. PHENIX Collaboration, A. Adare et al., Measurement of high$p_{t}$ single electrons from heavy-flavor decays in $\mathrm{p}+\mathrm{p}$ collisions at $\sqrt{s}=200 \mathrm{GeV}$. Phys. Rev. Lett. 97, 252,002 (2006). arXiv:hep-ex/0609010

45. STAR Collaboration, L. Adamczyk et al., Measurements of $D^{0}$ and $D^{*}$ production in p+p collisions at $\sqrt{s}=200 \mathrm{GeV}$. Phys. Rev. D 86, 072013 (2012). arXiv:1204.4244

46. E791 Collaboration, E.M. Aitala et al., Total forward and differential cross sections of neutral $D$ mesons produced in 500 $\mathrm{GeV} / \mathrm{c} \pi^{-}$-nucleon interactions. Phys. Lett. B 462, 225 (1999). arXiv:hep-ex/9906034

47. Beatrice Collaboration, M. Adamovich et al., Measurements of charmed-meson production in interactions between $350 \mathrm{GeV} / \mathrm{c} \pi^{-}$ particles and nuclei. Nucl. Phys. B 495, 3 (1997)

48. NA27 LEBC-EHS Collaboration, M. Aguilar-Benitez et al., Inclusive properties of $\mathrm{D}$ mesons produced in $360 \mathrm{GeV} \pi^{-} p$ interactions. Phys. Lett. B 161, 400 (1985)

49. G. Bari et al., A measurement of $\Lambda_{c}^{+}$baryon production in protonproton interactions at $\sqrt{s}=62 \mathrm{GeV}$. Nuovo Cim. A 104, 571 (1991)

50. R608 Collaboration, P. Chauvat et al., Production of $\Lambda_{c}$ with large $x_{F}$ at the ISR. Phys. Lett. B 199, 304 (1987)

51. LHCb Collaboration, R. Aaij et al., Measurements of prompt charm production cross-sections in $p p$ collisions at $\sqrt{s}=5 \mathrm{TeV}$. JHEP 06, 147 (2017). arXiv: 1610.02230

52. LHCb Collaboration, R. Aaij et al., Prompt charm production in $p p$ collisions at $\sqrt{s}=7 \mathrm{TeV}$. Nucl. Phys. B 871, 1 (2013). arXiv: 1302.2864

53. LHCb Collaboration, R. Aaij et al., Measurements of prompt charm production cross-sections in $p p$ collisions at $\sqrt{s}=13 \mathrm{TeV}$. JHEP 03, 159 (2016), Erratum: JHEP 05, 074 (2017). arXiv:1510.01707

54. S.I. Nikolsky, J.N. Stamenov, S.Z. Ushev, Composition of cosmic radiation at energies $\sim 10^{15} \mathrm{eV}$ and above. Sov. Phys. JETP 60, 10 (1984)

55. M. Thunman, G. Ingelman, P. Gondolo, Charm production and high-energy atmospheric muon and neutrino fluxes. Astropart. Phys. 5, 309 (1996). arXiv:hep-ph/9505417

56. T.K. Gaisser, Spectrum of cosmic-ray nucleons, kaon production, and the atmospheric muon charge ratio. Astropart. Phys. 35, 801 (2012). arXiv:1111.6675

57. IceCube Collaboration, M.G. Aartsen et al., Search for a diffuse flux of astrophysical muon neutrinos with the IceCube 59-string configuration. Phys. Rev. D 89, 062,007 (2014). arXiv:1311.7048

58. IceCube Collaboration, M.G. Aartsen et al., Atmospheric and astrophysical neutrinos above $1 \mathrm{TeV}$ interacting in IceCube. Phys. Rev. D 91, 022,001 (2015) 